\title{
Langevin Diffusion in Holographic Backgrounds with Hyperscaling Violation
}

\author{
J. Sadeghi and F. Pourasadollah \\ Department of Physics, Sciences Faculty, Mazandaran University, P.O. Box 47415-416, Babolsar, Iran \\ Correspondence should be addressed to J. Sadeghi; pouriya@ipm.ir
}

Received 18 May 2014; Accepted 9 September 2014; Published 20 October 2014

Academic Editor: Reinhard Schlickeiser

Copyright (c) 2014 J. Sadeghi and F. Pourasadollah. This is an open access article distributed under the Creative Commons Attribution License, which permits unrestricted use, distribution, and reproduction in any medium, provided the original work is properly cited. The publication of this article was funded by SCOAP ${ }^{3}$.

\begin{abstract}
We consider a relativistic heavy quark which moves in the quark-gluon plasmas. By using the holographic methods, we analyze the Langevin diffusion process of this relativistic heavy quark. This heavy quark is described by a trailing string attached to a flavor brane and moving at constant velocity. The fluctuations of this string are related to the thermal correlators and the correlation functions are precisely the kinds of objects that we compute in the gravity dual picture. We obtain the action of the trailing string in hyperscaling violation backgrounds and we then find the equations of motion. These equations lead us to constructing the Langevin correlator which helps us to obtain the Langevin constants. Using the Langevin correlators we derive the spectral densities and simple analytic expressions in the small- and large-frequency limits. We examine our works for planar and $R$-charged black holes with hyperscaling violation and find new constraints on $\theta$ in the presence of velocity $v$.
\end{abstract}

\section{Introduction}

It is certainly important and interesting to understand the strongly coupled quark-gluon plasma (QGP) [1-4], since heavy-ion collisions experiments have provided a variety of evidences for creation of QGPs at RHIC. Over the recent years, there have been a lot of efforts to study the features of heavy-ion collisions and the QGP. In this context, the AdS/CFT correspondence [5-8] has provided a powerful tool to study strongly coupled field theory. It maps relativistic conformal field theories holographically to gravitational (or stringy) dynamics in a higher dimensional spacetime. This gauge/gravity duality provides the possibility of computing some properties of QGP.

QGPs can be thought of as a soup of quarks and gluons. A heavy quark immersed in this fluid can be modeled in string theory (via the AdS/CFT correspondence) by an open string attached to the boundary of a bulk black hole. The endpoint of this string receives the heavy quark on a boundary which is stretching in the UV part of bulk geometry. At a classical level, the straight string is a solution to the equation of motion and does not move in the absence of external force. In this case, the string extends from the boundary to the black-hole horizon (at $r=r_{h}$ ). On the field theory side, a competition between the drag and the noise is balanced. And also the modes of string are in equilibrium at the Hawking temperature. The effect of thermal noise is not often considered in AdS/CFT. This seems to conflict with the fluctuation-dissipation theorem [9]. Clearly, one can expect that the Hawking radiation, which is emitted from the black brane, persuades the string to have a random motion. The fluctuations caused by the Hawking radiation are integrated within the stretched horizon $r_{s}=r_{h}+\epsilon$. This gives a picture of the stochastic behavior of the string fluctuations as originating from the world-sheet horizon with the required noise at this horizon [10]. The fluctuations of the trailing string (quantum) provide the information about the heavy quark as it moves in the plasma. So, the dynamics of fields on the boundary can be dictated by the effective action at the stretched horizon [11-13].

In this new scheme, in analogy with the dynamics of heavy quarks in heat bath giving rise to Brownian motion 
[14-20], one can consider the stochastic nature out of equilibrium systems. This involves a diffusive process that was first considered by using the Schwinger-Keldysh formalism adapted to AdS/CFT [21]. Another important improvement in this picture is related to relativistic Langevin evolution of the trailing string which is studied by [22-26]. The stochastic motion was formulated as a Langevin process [1116] associated with the correlators of the fluctuations of the string.

On the practical perspective, one can consider a fundamental string whose end-point lies in the UV region of a bulk black-hole background. The end-point of string is forced to move with velocity $v$. The string stretches in the bulk until the stretched horizon in this way becomes completely horizontal. When the quark is not moving (or moving with $v \rightarrow 0$ ) the stretched horizon approaches the black-hole horizon. The classical profile of the trailing string can be obtained by solving the Nambu-Goto equation of motion. By considering small fluctuations around the classical string profile at the quadratic level, second-order radial equations are obtained. These fluctuations are related to the thermal correlators with modified temperature $T_{s}$, through the second-order radial equations, and satisfy the fluctuation-dissipation relation associated with this temperature. Since in this case the system is out of equilibrium, the Hawking temperature of the induced string world-sheet metric $T_{s}$ is in general different from the heat bath temperature $T$. A relativistic Langevin diffusion equation is associated with the correlators of the fluctuations of the string by the holographic prescription. The Langevin correlators obey the modified Einstein relation with modified temperature $T_{s}$ and the relation between the diffusion constants is changed with this modified Einstein relation. $[27,28]$.

There is already a huge amount of literature on the subject of holographic construction of a heavy quark immersed in the quark-gluon plasma. The motion of such a quark has been studied holographically in the classical and relativistical way in $[16-20,27,28]$. In various articles the entries have been assigned to the investigation of sting fluctuations in the gravity theories where the corresponding plasmas have different features (e.g., rotation, charge, and ...) [18-20, 2933]. The construction of some holographic setups in the literature is formed so that the boundary theory is not conformally invariant. The holographic techniques have been used in the study of the submerging quark in such plasmas in $[27,28,33]$. The purpose of the present paper is to investigate the relativistic Langevin evolution of a heavy quark in backgrounds with hyperscaling violation [33-40].

Hyperscaling is a feature of the free energy based on naive dimension. For the theories with hyperscaling, the entropy behaves as $S \sim T^{d / z}$, where $T, d$, and $z$ are temperature, number of spatial dimensions, and dynamical exponent, respectively. Hyperscaling violation is first mentioned in context of holographic in [41]. In this context, the hyperscaling violation exponent $\theta$ is related to the transformation of the proper distance, and its noninvariance implies the violation of hyperscaling of the dual field theory. Then, the relation between the entropy and temperature has been modified as
$S \sim T^{(d-\theta) / z}$. In general, theory with hyperscaling violation $d-\theta$ plays the role of an effective space dimensionality for the dual field theory. In theories with hyperscaling violation, the metric backgrounds are the sophisticated generalization of the AdS gravity. These metrics have a special characteristic so that they can be dual to the field theories which are not conformally invariant. The observations $[34,35,42,43]$ indicate that backgrounds whose asymptotic behavior coincides with these metrics may be of interest to condensed matter physics. So, it is natural to further explore gauge/gravity duality for these backgrounds.

This paper is structured as follows. In Section 2 we present the description of backgrounds with hyperscaling violation and the relevant classical trailing string solution in these backgrounds. In Section 3 we carry out the corresponding linear fluctuations; these fluctuations are utilized for the holographic computation of the Langevin correlators. Also, we obtain the Langevin coefficients and the spectral density associated with the Langevin correlators. In Sections 2 and 3 our computations are devoted to the planar black holes with hyperscaling violation. But, in Section 4, we investigate the above-mentioned computations for $R$-charged black hole with hyperscaling violation. In Section 5, we summarize our works and make some comments for future research.

\section{Backgrounds with Hyperscaling Violation}

In this section, we implement the backgrounds with hyperscaling violation as the bulk geometries. The string extends into the bulk and is a dual of a heavy external quark moving through the plasma on the boundary of bulk. In order to discuss the Langevin coefficients of a heavy quark with gauge/gravity duality techniques, we have to find the fluctuations of the trailing string. These fluctuations are related to the thermal correlators and the correlation functions are precisely the kinds of objects that we compute in the gravity dual picture. Therefore we obtain the action of the trailing string in the backgrounds with hyperscaling violation and then we find the equations of motion from that action. By solving these equations we are able to find the Langevin correlator which helps us obtain the Langevin constants.

2.1. Planar Black Holes with Hyperscaling Violation. As we indicated before, we want to utilize backgrounds with hyperscaling violation as the bulk geometries. These geometries arise generically as the solutions in appropriate EinsteinMaxwell-dilaton theories with the following action [40, 41, 44],

$$
\begin{aligned}
S= & -\frac{1}{16 \pi G} \int d^{d+2} x \sqrt{-g} \\
& \times\left[\Re-\frac{1}{2}(\partial \phi)^{2}-f(\phi) F_{\mu \nu} F^{\mu \nu}+V(\phi)\right],
\end{aligned}
$$

where $\phi$ is dilaton field and the symbols $g$ and $\Re$ are determinant of the metric and the scalar curvature, respectively. The gauge coupling $g^{2}=(f(\phi))^{-1}$ and the potential $V(\phi)$ are both a function of the dilaton. The black-hole solution 
with hyperscaling violation from action (9) can be written as $[34,44]$

$$
\begin{gathered}
d s_{d+2}^{2} \\
=\left(\frac{R}{r}\right)^{2}\left(\frac{r}{r_{F}}\right)^{2 \theta / d}\left[-r^{-2(z-1)} h(r) d t^{2}+h(r)^{-1} d r^{2}+d x_{i}^{2}\right], \\
h(r)=1-\left(\frac{r}{r_{h}}\right)^{d+z-\theta} .
\end{gathered}
$$

We note here that the metric background includes a dynamical critical exponent $z$ and a hyperscaling violation exponent $\theta$; also $d$ is the number of transverse dimensions and $i=$ $1, \ldots, d, r=r_{h}$, is the location of horizon and $r=r_{F}$ is the boundary. This metric is not scale invariant under the following scaling:

$$
t \longrightarrow \lambda^{z} t, \quad x_{i} \longrightarrow \lambda x_{i}, \quad r \longrightarrow \lambda r
$$

and transforms as

$$
d s \longrightarrow \lambda^{\theta / d} d s,
$$

which is defining property of hyperscaling in holographic language. In order to understand the metric properties of this class of spacetimes, notice that (2) is conformally equivalent to a Lifshitz geometry $[45,46]$ as can be seen after a Weyl rescaling $g_{\mu \nu} \rightarrow \widetilde{g}_{\mu \nu}=\Omega^{2} g_{\mu \nu}$, with $\Omega=r^{-(\theta / d)}$. The scaleinvariant limit is $\theta=0$, which reduces to a Lifshitz solution.

It is reasonable from the gravity side to demand that the null energy condition (NEC) $[34,40]$ be satisfied. For metric background (2), this imposes some constraints on $\theta$ and $z$ as

$$
(d-\theta)(d(z-1)-\theta) \geq 0, \quad(z-1)(d+z-\theta) \geq 0 .
$$

These constraints have important consequences. First, in a Lorentz invariant theory, $z=1$ and then the first inequality implies that $\theta \leq 0$ or $\theta \geq d$. On the other hand, for a scaleinvariant theory $(\theta=0)$, we recover the known result $z \geq 1$. Notice that in theories with hyperscaling violation the NEC can be satisfied for $z<1$, while this range of dynamical exponents is forbidden if $\theta=0$. In particular, $\{z<0 ; \theta>d\}$ gives a consistent solution to (5), as well as $\{0<z<1$; $\theta \geq$ $d+z$ \}. The NEC gives $\theta>d$, but this range for $\theta$ leads to instabilities in the gravity side. So this choice of $\theta$ does not lead to the physically consistent theories.

2.2. Trailing String and Drag Force. Before going in detail we should indicate that we review the calculation of the unperturbed trailing solution that was discussed in $[27,28]$. We consider an external heavy quark which moves in the quark-gluon plasma medium with a fixed velocity $v$ on the boundary theory. It can be realized as the end-point of an open classical trailing string which is hanged from the boundary and moves at constant velocity $v$. The dynamics of this string is governed by the Nambu-Goto action,

$$
S_{\mathrm{NG}}=-\frac{1}{2 \pi \alpha^{\prime}} \int d \tau d \sigma \sqrt{-\operatorname{det} g_{a b}},
$$

where $g_{a b}=G_{\mu \nu} \partial_{a} X^{\mu} \partial_{b} X^{\nu}$ denotes the components of the bulk metric in the string frame. We choose the $\tau=t$ and $\sigma=r$ to work in static gauge and take the following ansatz for trailing string:

$$
X^{1}=v t+\xi(r), \quad X^{2}, X^{3}, \ldots, X^{d}=0 .
$$

By using the metric background (2), the induced metric on the world-sheet can be obtained as

$$
g_{a b}=\left(\frac{R}{r}\right)^{2}\left(\frac{r}{r_{F}}\right)^{2 \theta / d}\left(\begin{array}{cc}
v^{2}-r^{-2(z-1)} h(r) & v \xi^{\prime}(r) \\
v \xi^{\prime}(r) & h(r)^{-1}+\xi^{\prime 2}
\end{array}\right) .
$$

So, the corresponding action becomes

$$
\begin{aligned}
S_{\mathrm{NG}}= & -\frac{1}{2 \pi \alpha^{\prime}} \int d t d r\left(\frac{R}{r}\right)^{2}\left(\frac{r}{r_{F}}\right)^{2 \theta / d} \\
& \times \sqrt{r^{-2(z-1)}-\frac{v^{2}}{h(r)}+r^{-2(z-1)} h(r) \xi^{\prime 2}(r)} .
\end{aligned}
$$

The conjugate momentum $\pi_{\xi}$ that flowed from the boundary to the bulk and interpreted as the total force experienced by the quark is written by

$$
\xi^{\prime 2}=\frac{\left(v^{2}-r^{-2(z-1)} h(r)\right) C^{2}}{\left[C^{2}-(R / r)^{4}\left(r / r_{F}\right)^{4 \theta / d} r^{-2(z-1)} h(r)\right] r^{-2(z-1)} h^{2}(r)},
$$

through the relation $C=2 \pi \alpha^{\prime} \pi_{\xi}$. The horizon for the induced world-sheet metric is obtained:

$$
r_{s}^{-2(z-1)} h\left(r_{s}\right)=v^{2} .
$$

Since the numerator of the square root in (10) vanishes at the stretched horizon, reality requires that the denominator also vanishes. So, we have $\pi_{\xi}^{2}=\left(R / r_{s}\right)^{4}\left(r_{s} / r_{F}\right)^{4 \theta / d} r_{s}^{-2(z-1)}$ $\left(h\left(r_{s}\right) / 4 \pi^{2} \alpha^{\prime 2}\right)$. For $z=1$ the stretched horizon is given by

$$
r_{s}=r_{h}\left(1-v^{2}\right)^{1 /(d+1-\theta)} .
$$

In the special case $\theta=d+1$ the stretched horizon in (12) tends to the infinity. It is an unacceptable case, since we expect that the stretched horizon is smaller than the horizon $r_{h}$. Moreover, we eliminate $\theta>d+1$ for the similar reason.

The drag force on the quark can be obtained from the momentum conjugate which has the following form:

$$
\begin{aligned}
& F_{\text {drag }}=\pi_{\xi}=-\frac{v R^{2} r_{s}^{(2 \theta / d)-2}}{2 \pi \alpha^{\prime} r_{F}^{2(\theta / d)}}, \\
& F_{\text {drag }}=-\frac{R^{2} r_{h}^{2((\theta / d)-1)} v\left(1-v^{2}\right)^{2((\theta / d)-1) /(d+1-\theta)}}{2 \pi \alpha^{\prime} r_{F}^{2(\theta / d)}},
\end{aligned}
$$

$$
\text { for } z=1 \text {. }
$$


In the ultrarelativistic limit $v \rightarrow 1$ the drag force for the case $z=1$ vanishes, but one can check that this event does not happen for the range of $z>1$. The momentum friction coefficient $\eta$ is responsible for the gradual loss in the momentum of a quark of mass $M$. It is related to the drag force via the $F_{\text {drag }}=-\eta p$, with $p=M \gamma v$ [47]. So, one can obtain

$$
\eta=\frac{R^{2} r_{h}^{2((\theta / d)-1)}\left(1-v^{2}\right)^{2((\theta / d)-1) /(d+1-\theta)}}{2 \pi \alpha^{\prime} M \gamma r_{F}^{2(\theta / d)}}
$$

where $\gamma=1 / \sqrt{1-v^{2}}$ is the relativistic contraction factor. For the special case $\theta=d$, the drag force and momentum friction coefficient reduce to the following expression:

$$
F_{\text {drag }}=-\frac{R^{2} v}{2 \pi \alpha^{\prime} r_{F}^{2}}, \quad \eta=\frac{R^{2}}{2 \pi \alpha^{\prime} M \gamma r_{F}^{2}} .
$$

In this case, $F_{\text {drag }}$ and $\eta$ are independent of the black-hole horizon.

If we diagonalize the induced world-sheet metric by transforming the coordinate through the transformation $d t \rightarrow d t-\left(v \xi^{\prime}(r) /\left(v^{2}-r^{-2(z-1)} h(r)\right)\right) d r$, the resulting metric components are

$$
\begin{gathered}
h_{t t}=\left(\frac{R}{r}\right)^{2}\left(\frac{r}{r_{F}}\right)^{2 \theta / d}\left(-r^{-2(z-1)} h(r)+v^{2}\right), \\
h_{r r}=\frac{r^{-2(z-1)}(R / r)^{6}\left(r / r_{F}\right)^{6 \theta / d}}{r^{-2(z-1)}(R / r)^{4}\left(r / r_{F}\right)^{4 \theta / d} h(r)-C^{2}} .
\end{gathered}
$$

The effective Hawking temperature associated with the above black-hole metric can be found:

$$
\begin{aligned}
T_{s}^{2}=\frac{1}{16 \pi^{2}}[ & r_{s}^{-2(z-1)} h^{2}\left(r_{s}\right)\left(\frac{h^{\prime}\left(r_{s}\right)}{h\left(r_{s}\right)}+\frac{((4 \theta / d)-2(z+1))}{r_{s}}\right) \\
& \left.\times\left(\frac{h^{\prime}\left(r_{s}\right)}{h\left(r_{s}\right)}+\frac{-2(z-1)}{r_{s}}\right)\right] .
\end{aligned}
$$

For $z=1$, the above relation reduces to

$$
\begin{aligned}
& T_{s} \\
& =\frac{1}{4 \pi} \\
& \quad \times\left(\left(\left[\left(\frac{4 \theta}{d}+d-3-\theta\right)\left(1-v^{2}\right)-\left(\frac{4 \theta}{d}-4\right)\right]\right.\right. \\
& \left.\left.\quad \times\left(1-v^{2}\right)(d+1-\theta)\right) \times\left[r_{h}\left(1-v^{2}\right)^{1 /(d+1-\theta)}\right]^{-2}\right)^{1 / 2} .
\end{aligned}
$$

In order to have correct value for $T_{s}$ in the case of $\theta<d+1$, we need to have the following condition:

$$
\theta<\frac{d\left[1+d+(3-d) v^{2}\right]}{d+(4-d) v^{2}}
$$

In the special case $d=3$, we have

$$
\theta<\frac{12}{3+v^{2}}
$$

The Hawking temperature related to the black-hole horizon in the presence of hyperscaling parameter and dynamical exponent $z=1$ is defined as

$$
T=\frac{(d+1-\theta)}{4 \pi r_{h}}
$$

From (18) and (21), one can easily find that

$$
T_{s}^{2}=T^{2}\left[1-\frac{((4 \theta / d)+d-3-\theta) v^{2}}{(d+1-\theta)}\right]\left(1-v^{2}\right)^{1-(2 /(d+1-\theta))}
$$

For the special case $\theta=d$, it is obvious from the above relation that the Hawking temperature and the modified temperature become equal. This equality is also confirmed for the range $z>1$. In the conformal limit, it means the hyperscaling parameter tends to zero and the background solution reduces to AdS-Schwarzschild. So, the stretched horizon position and temperature are given by

$$
\begin{gathered}
T_{s}^{2}=T^{2}\left[1-\frac{d-3}{d+1} v^{2}\right]\left(1-v^{2}\right)^{1-(2 /(d+1))}, \\
r_{s}=\frac{d+1}{4 \pi T}\left(1-v^{2}\right)^{1 /(d+1)} .
\end{gathered}
$$

For $d=3$ we receive the expected relation [28]

$$
T_{s}=\frac{T}{\sqrt{\gamma}}, \quad r_{s}=\frac{1}{4 \pi \sqrt{\gamma} T}
$$

2.3. Fluctuations of the Trailing String. In order to study the stochastic motion of quark, we proceed to investigate the fluctuations around the classical trailing solution. We choose the static gauge, such that the string embedding becomes $X^{\mu}(t, r)=\left(t, r, X^{1}(t, r), X^{2}(t, r), \ldots, X^{d}(t, r)\right)$. So, we take the following ansatz for embedding:

$$
\begin{gathered}
X^{1}(t, r)=v t+\xi(r)+\delta X^{\|}(t, r), \\
X^{2}(t, r)=\delta X^{2}(t, r), \ldots, X^{d}(t, r)=\delta X^{d}(t, r) .
\end{gathered}
$$

By expanding the Nambu-Goto action in $\delta X^{i}(t, r)$ around the classical solution up to quadratic terms we have

$$
\begin{aligned}
S_{2}= & -\frac{1}{2 \pi \alpha^{\prime}} \int d t d r \frac{H^{a b}}{2} \\
& \times\left[N(r) \partial_{a} \delta X^{\|} \partial_{b} \delta X^{\|}+\sum_{i=2}^{d} G_{i i} \partial_{a} \delta X^{i} \partial_{b} \delta X^{i}\right],
\end{aligned}
$$


where

$$
\begin{gathered}
N(r)=\frac{\left(r^{-2(z-1)} h(r)(R / r)\right)^{4}\left(r / r_{F}\right)^{4 \theta / d}-C^{2}}{(R / r)^{2}\left(r / r_{F}\right)^{2 \theta / d}\left(r^{-2(z-1)} h(r)-v^{2}\right)}, \\
G_{i i}=\left(\frac{R}{r}\right)^{4}\left(\frac{r}{r_{F}}\right)^{4 \theta / d},
\end{gathered}
$$

and $H^{a b}=\sqrt{-h} h^{a b}$. The equations of motion can be found from the above action

$$
\partial_{a}\left(H^{a b} N(r) \partial_{b} \delta X^{\|}\right)=0, \quad \partial_{a}\left(H^{a b} G_{i j} \partial_{b} \delta X^{\perp}\right)=0 .
$$

The definitions of $\delta X^{\perp}$ and $\delta X^{\|}$are responsible for the longitudinal and the transverse fluctuations, respectively. By taking a harmonic ansatz as $\delta X^{i}(r, t)=e^{i \omega t} \delta X^{i}(r, \omega),(28)$ become

$$
\begin{gathered}
\partial_{r}\left[\frac{\sqrt{\left(C^{2}-(R / r)^{4}\left(r / r_{F}\right)^{4 \theta / d} r^{-2(z-1)} h(r)\right)\left(v^{2}-r^{-2(z-1)} h(r)\right)}}{r^{-(z-1)}} \partial_{r} \delta X^{\perp}\right] \\
+\frac{\omega^{2} r^{-(z-1)}(R / r)^{4}\left(r / r_{F}\right)^{4 \theta / d}}{\sqrt{\left(C^{2}-(R / r)^{4}\left(r / r_{F}\right)^{4 \theta / d} r^{-2(z-1)} h(r)\right)\left(v^{2}-r^{-2(z-1)} h(r)\right)}} \delta X^{\perp}=0, \\
\partial_{r}\left[\frac{\left(C^{2}-(R / r)^{4}\left(r / r_{F}\right)^{4 \theta / d} r^{-2(z-1)} h(r)\right)^{3 / 2}}{r^{-(z-1)}\left(v^{2}-r^{-2(z-1)} h(r)\right)^{1 / 2}} \partial_{r} \delta X^{\|}\right]+\frac{\omega^{2} r^{-(z-1)}\left(C^{2}-(R / r)^{4}\left(r / r_{F}\right)^{4 \theta / d} r^{-2(z-1)} h(r)\right)^{1 / 2}}{\left(v^{2}-r^{-2(z-1)} h(r)\right)^{3 / 2}} \delta X^{\|}=0 .
\end{gathered}
$$

In what follows, we will construct solutions to (29) for the string fluctuations and obtain the diffusion constants and the spectral density from them. However, by using the method of the membrane paradigm, computation of diffusion constants can be done directly from the quadratic action (26). We will derive these constants through the two different methods in the next section.

\section{Holographic Computation of Langevin Correlators and Diffusion Constants}

3.1. Momentum Correlators from the Trailing String. The Langevin correlators can be computed holographically from the classical solutions for the fluctuations of the trailing string. Two types of independent retarded correlators $g_{R}^{\|}$and $g_{R}^{\perp}$ for the longitudinal and transverse fluctuations [22] are reasonably expected from the structure of action (26) for the fluctuations

$$
g_{\|}^{a b}=\frac{N}{2 \pi \alpha^{\prime}} H^{a b}, \quad g_{\perp}^{a b}=\frac{G_{i i}}{2 \pi \alpha^{\prime}} H^{a b} .
$$

In the holographic version for the retarded correlator of diagonal metric (16) we have

$$
G_{R}(\omega)=-\left[\psi^{*}(r, \omega) g^{r r} \partial_{r} \psi(r, \omega)\right]_{\text {boundary }}
$$

where $\psi$ is related to the fluctuations $\delta X^{\|}$and $\delta X^{\perp}$. The expression in (31) must be evaluated at the boundary of the trailing string world-sheet. In theories with hyperscaling violation, existence of a dimensionful scale that does not decouple in the infrared requires the proper powers of this scale, which is denoted by $r_{F}$. Also we note here, by following the effective holographic approach [48] in which the dual theory lives on a finite $r$ slice, the metric background (2) provides a good description of the dual field theory only for a certain range of $r$, presumably for $r \geq r_{F}$ anticipating the applications at the low energy regions. In the case of an infinitely massive quark, the string is attached at the AdS boundary at $r=0$; however, in our case with hyperscaling violation, the string is connected to the boundary at $r=r_{F}$, where $r_{F} \rightarrow 0$. In the case of finite mass quark, the trailing string is attached to a point $r_{b}$ and its mass is given through the following relation:

$$
\begin{aligned}
M & =\frac{1}{2 \pi \alpha^{\prime}} \int_{0}^{r_{b}}\left(\frac{R}{r_{F}^{\theta / d}}\right)^{2} r^{(2 \theta / d)-2-z+1} \\
& =\frac{1}{2 \pi \alpha^{\prime}}\left(\frac{R}{r_{F}^{\theta / d}}\right)^{2} \frac{r_{b}^{(2 \theta / d)-z}}{(2 \theta / d)-z} .
\end{aligned}
$$

For $\theta \sim(z / 2) d$, the mass of quark tends to the infinity. Moreover, we expect an infinitely massive quark for $r_{b} \rightarrow 0$. Thus, $\theta<(z / 2) d$ is the acceptable region for $\theta$ to make the expected mass for quark in the limit $r_{b} \rightarrow 0$ [49].

The solutions of fluctuation equations (29) have the same behavior for the transverse and longitudinal components at 
the world-sheet horizon and at the boundary. At the $r \rightarrow r_{s}$ limit both equations take following form,

$$
\partial_{r}^{2} \psi+\frac{1}{\left|r-r_{s}\right|} \partial_{r} \psi+\left(\frac{\omega}{4 \pi T_{s}\left|r-r_{s}\right|}\right)^{2} \psi=0
$$

so, the solution in near world-sheet horizon is given by

$$
\psi(r, \omega) \sim\left(r_{s}-r\right)^{ \pm i\left(\omega / 4 \pi T_{s}\right)}
$$

where $T_{s}$ is the modified temperature which is given by (17) and (18) for special case $(z=1)$. In the above relation the outgoing waves are brought in + sign while the incoming waves are in - sign.

Near the boundary $r \rightarrow r_{F}$, for (29), we have to discuss the range of $\theta$ and $z$. Let us consider $\theta<d+z$, where $h(r)$ vanishes in the limit of $r \rightarrow r_{F} \rightarrow 0$. In the special case $z=1$ both equations reduce to

$$
\partial_{r}^{2} \psi+\frac{(2 \theta / d)-2}{r} \partial_{r} \psi+\omega^{2} \gamma^{2} \psi=0
$$

therefore the solution of above equation is given by

$$
\begin{aligned}
\psi= & c_{1} r^{(3 d-2 \theta) / 2 d} J_{-(3 d-2 \theta) / 2 d}(\gamma \omega r) \\
& +c_{2} r^{(3 d-2 \theta) / 2 d} Y_{-(3 d-2 \theta) / 2 d}(\gamma \omega r),
\end{aligned}
$$

where for small $\omega$ two independent solutions with normalizable and nonnormalizable modes become

$$
\psi=c_{s}+c_{v} r^{(3 d-2 \theta) / d} .
$$

Near the boundary for the range of $z>0$, we have

$$
\begin{aligned}
\mathbf{U} & =C^{2}-\left(\frac{R}{r}\right)^{4}\left(\frac{r}{r_{F}}\right)^{4 \theta / d} r^{-2(z-1)} h(r) \longrightarrow-\frac{R^{4}}{r_{F}^{2(z+1)}}, \\
\mathbf{V} & =v^{2}-r^{-2(z-1)} h(r) \longrightarrow v^{2} \quad \text { for } z<1, \\
& \longrightarrow-r_{F}^{-2(z-1)} \quad \text { for } z>1 .
\end{aligned}
$$

Then, fluctuation equations (29) for $z>1$ near the boundary for both cases become

$$
\partial_{r}^{2} \psi+\frac{(2 \theta / d)-1-z}{r} \partial_{r} \psi+\omega^{2} r^{2(z-1)} \psi=0,
$$

which has the following solutions:

$$
\begin{aligned}
\psi= & c_{1} r^{((z+2) d-2 \theta) / 2 d} J_{-((z+2) d-2 \theta) / 2 z d}\left(\frac{\gamma \omega}{z} r^{z}\right) \\
& +c_{2} r^{((z+2) d-2 \theta) / 2 d} Y_{-((z+2) d-2 \theta) / 2 z d}\left(\frac{\gamma \omega}{z} r^{z}\right) .
\end{aligned}
$$

Also, in the region $\omega r \ll 1$ we receive the following equation:

$$
\psi=c_{s}+c_{v} r^{(2 d+z d-2 \theta) / d}
$$

For $z<1$ these equations reduce to

$$
\partial_{r}^{2} \psi+\frac{(2 \theta / d)-2}{r} \partial_{r} \psi-\frac{\gamma^{2}}{v^{2}} \psi=0
$$

and the solution of above equation will be

$$
\begin{aligned}
\psi= & c_{1} r^{(3 d-2 \theta) / 2 d} I_{-((3 d-2 \theta) / 2 d)}\left(\frac{\omega}{v} r\right) \\
& +c_{2} r^{(3 d-2 \theta) / 2 d} K_{-((3 d-2 \theta) / 2 d)}\left(\frac{\omega}{v} r\right),
\end{aligned}
$$

and for the small $\omega$ we receive relation (37).

The appropriate boundary conditions for the wavefunctions in the expression (34) for the retarded correlator are in-falling behavior at the world-sheet horizon with the condition $\psi(r)=1[50]$ :

$$
\begin{aligned}
& \psi\left(r_{b}, \omega\right)=1 \quad r_{b}= \begin{cases}0 & M \longrightarrow \infty \\
r_{q} & M \text { finite },\end{cases} \\
& \psi(r, \omega) \sim \psi_{h}\left(r_{s}-r\right)^{-i\left(\omega / 4 \pi T_{s}\right)} \quad r \sim r_{s} .
\end{aligned}
$$

By utilizing the wave-functions, we can construct the propagator from relation (31). We consider the properties of real and imaginary parts of retarded Green's functions separately.

Real Part of Retarded Correlator. In the real part of retarded correlator (31) there are some ambiguities related to the UV divergencies in the on-shell action. To avoid these divergencies and receive finite results, one has to investigate the action on a regularized spacetime with boundary at $r=$ $r_{F}$. Then, after identifying the divergencies in the limit $r_{F} \rightarrow$ 0 , the counterterms can be added to prevent infinite results.

To evaluate the real part of (31), the wave-functions close to the boundary can be implemented for different regions of $z$. For $z=1$, we can expand the solution (36) for $\gamma \omega r \ll 1$ near the boundary $r=r_{F}$ as

$$
\begin{aligned}
\psi_{\mathrm{UV}}(r) & \\
= & c_{1}\left(\frac{\gamma \omega}{2}\right)^{(-3 d+2 \theta) / 2 d} \\
& \times\left[\frac{1}{\Gamma((-1 / 2)+(\theta / d))}-\frac{(\gamma \omega)^{2} r_{F}^{2}}{4 \Gamma((1 / 2)+(\theta / d))}\right] \\
& -\frac{c_{2}}{\pi}\left(\frac{\gamma \omega}{2}\right)^{(3 d-2 \theta) / 2 d} r_{F}^{(3 d-2 \theta) / d} \\
& \times\left[\Gamma\left(\frac{-3}{2}+\frac{\theta}{d}\right)+\frac{\Gamma((-5 / 2)+(\theta / d))(\gamma \omega)^{2} r_{F}^{2}}{4}\right]+\cdots
\end{aligned}
$$


According to relation (44) the value of $c_{1}=c_{s}$ is fixed at $r=r_{F}$ :

$$
c_{1}=c_{s}=\left(\frac{\gamma \omega}{2}\right)^{(3 d-2 \theta) / 2 d} \Gamma\left(\frac{-1}{2}+\frac{\theta}{d}\right) .
$$

To obtain the real part of (31) we must evaluate relation (30) at the boundary; then we have

$$
H^{r r} \sim \frac{1}{\gamma}, \quad H^{t t} \sim-\gamma, \quad N \sim \frac{\gamma^{2} R^{2}}{r_{F}^{2}} .
$$

Eventually, we find the following divergent term from the expression (46) for the transverse and longitudinal components:

$$
\begin{aligned}
\operatorname{Re} G_{R}^{\perp} \simeq & \gamma^{-2} \operatorname{Re} G_{R}^{\|} \simeq \frac{\gamma \omega^{2}}{2 \pi \alpha^{\prime}(1-(2 \theta / d)) r_{F}} \\
& \times\left[1+\frac{\gamma^{2} \omega^{2} r_{F}^{2}}{2(1-(2 \theta / d))}+O\left(r_{F}^{4}\right)\right] .
\end{aligned}
$$

Notice that in derivation of the above relation we neglect the effect of the second term in (46), which is proportional to $c_{2}=$ $c_{v}$ and starts at $O\left(r_{F}^{3-(2 \theta / d)} \omega^{(3 d-2 \theta) / 2 d}\right)$ (considering the region of $\theta<d+1$ ). The correlators have UV divergences (in the $\omega^{2}$ term) which arise from the scheme dependence in their calculation.

We now address the analysis of on-shell action to obtain the transverse and longitudinal Green's functions and compare them with the results of relation (49). We study the divergence structure of action (6), expanded to quadratic order in the fluctuations defined in (26), around the classical trailing string solution [28]. So we write

$$
S_{\mathrm{NG}}=S_{0}+S_{1}+S_{2}+\cdots \text {. }
$$

For each term in the above relation we derive the divergency around $r=r_{F}$ separately. The zeroth order term reads simply:

$$
S_{0}=\frac{-1}{2 \pi \alpha^{\prime}} \int d t d r \sqrt{-g}=\frac{-1}{2 \pi \alpha^{\prime}} \int d t \int_{r_{F}}^{r_{s}} d r \frac{R^{2}}{\gamma r_{F}^{2 \theta / d}} r^{(2 \theta / d)-2} .
$$

Around $r=r_{F}$, the above integral shows a divergency of the order $1 / r_{F}$ :

$$
S_{0}^{\mathrm{div}}=\frac{R^{2}}{2 \pi \alpha^{\prime}(1-(2 \theta / d)) \gamma r_{F}} \int d t
$$

For the second term in (30) the quadratic order action (26) is implemented. By inserting the solution (37) close to the boundary and using relation (48), we obtain the divergent part of (26) as

$$
\begin{aligned}
S_{2}^{\mathrm{div}}= & \frac{R^{2}}{2 \pi \alpha^{\prime}(1-(2 \theta / d)) \gamma r_{F}} \frac{1}{2} \\
& \times \int d \omega \omega^{2}\left(\gamma^{2}\left|c_{s}^{\perp}(\omega)\right|^{2}+\gamma^{4}\left|c_{s}^{\|}(\omega)\right|^{2}\right) \\
= & \frac{R^{2}}{2 \pi \alpha^{\prime}(1-(2 \theta / d)) \gamma r_{F}} \frac{1}{2} \int d t \gamma^{2}\left(\delta \dot{X}^{\perp}\right)^{2}+\gamma^{4}\left(\delta \dot{X}^{\|}\right)^{2} .
\end{aligned}
$$

One can easily check that there is no divergency coming from the first-order action. Consequently, from the above action the divergent parts of the transverse and longitudinal Green's functions are

$$
\begin{aligned}
& \left(\operatorname{Re} G_{R}^{\perp}\right)=\frac{R^{2}}{2 \pi \alpha^{\prime}(1-(2 \theta / d)) r_{F}} \gamma \omega^{2}, \\
& \left(\operatorname{Re} G_{R}^{\|}\right)=\frac{R^{2}}{2 \pi \alpha^{\prime}(1-(2 \theta / d)) r_{F}} \gamma^{3} \omega^{2} .
\end{aligned}
$$

The resorption of both (52) and (53) divergencies can be done by adding a single covariant boundary counterterm,

$$
S_{\text {count }}=\Delta M\left(r_{F}\right) \int d t \sqrt{\dot{X}^{\mu} \dot{X}_{\mu}}
$$

which is responsible for the renormalization of the quark mass. By expanding the above relation to the second order in $\vec{X}=\vec{v} t+\delta \vec{X}$, we find

$$
S_{\text {count }}=\frac{\Delta M\left(r_{F}\right)}{\gamma}\left\{\int d t+\int \frac{1}{2} \int d t \gamma^{2}\left(\delta \dot{X}^{\perp}\right)^{2}+\gamma^{4}\left(\delta \dot{X}^{\|}\right)^{2}\right\} .
$$

It is clear from (52) and (53) that the following choice for $\Delta M$ eliminates the total divergencies:

$$
\Delta M\left(r_{F}\right)=-\frac{R^{2}}{2 \pi \alpha^{\prime}(1-(2 \theta / d)) r_{F}}
$$

If we repeat the above progress for the case of $z>1$ for the zeroth order we have

$$
S_{0}^{\operatorname{div}}=\frac{R^{2}}{2 \pi \alpha^{\prime}(z-(2 \theta / d)) r_{F}^{z}} \int d t
$$

The divergency coming from the second-order action is given by

$$
\begin{aligned}
S_{2}^{\text {div }}= & \frac{R^{2}}{2 \pi \alpha^{\prime}(2-z-(2 \theta / d)) r_{F}^{2-z}} \frac{1}{2} \\
& \times \int d \omega \omega^{2}\left(\left|c_{s}^{\perp}(\omega)\right|^{2}+\left|c_{s}^{\|}(\omega)\right|^{2}\right) \\
= & \frac{R^{2}}{2 \pi \alpha^{\prime}(2-z-(2 \theta / d)) r_{F}^{2-z}} \frac{1}{2} \int d t\left(\delta \dot{X}^{\perp}\right)^{2}+\left(\delta \dot{X}^{\|}\right)^{2} .
\end{aligned}
$$


So the divergent parts of the transverse and longitudinal Green's functions are identical as

$$
\left(\operatorname{Re} G_{R}^{\perp}\right)=\left(\operatorname{Re} G_{R}^{\|}\right)=\frac{R^{2}}{2 \pi \alpha^{\prime}(2-z-(2 \theta / d)) r_{F}^{2-z}} \omega^{2},
$$

where this result can also explicitly be derived from the explicit expansions of the wave-functions (40) close to the boundary. We note that, to get the result of (59), we use the following relation:

$$
H^{r r} \sim r_{F}^{-(z-1)}, \quad H^{t t} \sim-r_{F}^{z-1}, \quad N \sim \frac{\gamma^{2} R^{2}}{r_{F}^{2}}
$$

By comparing two divergencies in the zeroth and the second order, we find that the zeroth order divergency dominates over the second order since $z>1$. For the second order, the divergencies appear only in the range of $1<z<2$. So we may consider the following term for renormalizing the quark mass and fixing the coefficient of the counterterm action:

$$
\Delta M\left(r_{F}\right)=-\frac{R^{2} \gamma}{2 \pi \alpha^{\prime}(z-(2 \theta / d)) r_{F}^{z}} .
$$

Unfortunately, this choice for changes in the quark mass does not completely cancel divergencies coming from the on-shell action. It seems that the other counterterms should be added before cancelling these divergencies. However, we showed that the real parts of retarded correlator are equal to divergencies which come from the unrenormalized on-shell action. The discussion on removing the divergencies remains as a open problem we may investigate in the future.

In the region of $z<1$ all computations are similar to the case of $z=1$ except that in this region we must replace $\gamma$ with $1 / \sqrt{-v^{2}}$. This means that in the renormalization of quark mass we have to add a virtual mass to the quark to receive the finite results and also we encountered an imaginary value in the computation of the real part of retarded correlator. This seems somewhat complicated, but as we know this range of dynamical exponents is forbidden if $\theta=0[51,52]$. In particular, as discussed before the range of $0<z<1$ with $\theta \geq d+z$ gives a consistent solution to the null energy condition and to $z<0, \theta>0$. So one can conclude that the range of $0<z<1$ is not in agreement with $\theta<d+z$ (the range of $\theta$ that we assumed first at our work). In continuous paper, we do not consider this region for $z$.

Imaginary Part of Retarded Correlator. In the imaginary part of retarded correlator we do not encounter the divergencies, since it is proportional to the conserved quantity (current) as

$$
\operatorname{Im} G_{R}(\omega)=-\frac{1}{2 i} g^{r r} \psi_{R}^{*} \partial_{r} \psi_{R} \equiv-J^{r}
$$

and compute at the horizon. From definitions (27) and (30), we find, in the near-horizon limit,

$$
\begin{array}{r}
g_{\perp}^{r r} \simeq 4 \pi T_{s}\left(r_{s}-r\right) G_{i i}\left(r_{s}\right), \quad g_{\|}^{r r} \simeq 4 \pi T_{s}\left(r_{s}-r\right) N\left(r_{s}\right), \\
r \longrightarrow r_{s},
\end{array}
$$

where

$$
\begin{aligned}
N\left(r_{s}\right)= & \frac{16 \pi^{2} R^{2} T_{s}^{2}}{r_{F}^{2 \theta / d} h^{2}\left(r_{s}\right)\left(\left(h^{\prime}\left(r_{s}\right) / h\left(r_{s}\right)\right)+\left(-2(z-1) / r_{s}\right)\right)^{2}} \\
& \times r_{s}^{(2 \theta / d)+2 z-4} .
\end{aligned}
$$

By substituting the above relation in (63) and utilizing the solution (45) for $\psi$ one can write

$$
\operatorname{Im} G_{R}^{\perp}=-\frac{G_{i i}\left(r_{s}\right)\left|\psi_{h}^{\perp}\right|^{2}}{2 \pi \alpha^{\prime}} \omega, \quad \operatorname{Im} G_{R}^{\|}=-\frac{N\left(r_{s}\right)\left|\psi_{h}^{\|}\right|^{2}}{2 \pi \alpha^{\prime}} \omega
$$

From $[11,12,53,54]$ we get the imaginary part of the retarded correlator which is given by

$$
G(\omega)=\cot \left(\frac{\omega}{\left(2 T_{s}\right)}\right) \operatorname{Im} G_{R}(\omega)
$$

where $G_{R}(\omega)$ is symmetrized correlator. The spectral density associated with Langevin dynamics are defined as

$$
\begin{gathered}
\rho_{R}(\omega)=\frac{-1}{\pi} \operatorname{Im} G_{R}(\omega), \\
\rho(\omega)=\frac{-1}{\pi} \operatorname{Im} G(\omega)=\cot \left(\frac{\omega}{\left(2 T_{s}\right)}\right) \rho_{R}(\omega) .
\end{gathered}
$$

The above equations help us to investigate the spectral density at the large frequency. In order to study the behavior of Langevin correlators at the high frequency we have to use the WKB method.

3.2. The $W K B$ Approximation at Large Frequency. In this section we are going to derive the large-frequency limit of the spectral density, so in order to do this process we need to apply the WKB method. So, in order to arrange the equation as Schrödinger-like form (29) equations, we have to rescale the corresponding wave-function. The large $\omega$ solution can be obtained by an adaptation of the WKB method $[28,55]$. The Schrödinger-like form of this equations is

$$
\begin{gathered}
-\phi^{\prime \prime}+V_{s}(r) \phi=0, \\
V_{s}(r)=-\frac{\omega^{2} G_{i i}^{2}}{L^{2}}+\frac{1}{2}(\log \mathscr{L})^{\prime \prime}+\frac{1}{4}(\log \mathscr{L})^{\prime 2},
\end{gathered}
$$


where $\phi=\sqrt{\mathscr{L}} \psi$ with

$$
\psi=\left\{\begin{array}{l}
\delta X^{\perp} \\
\delta X^{\|},
\end{array} \quad \mathscr{L}=\left\{\begin{array}{l}
L \\
\frac{L N}{G_{i i}},
\end{array}\right.\right.
$$

$L$

$$
=\frac{\sqrt{\left(C^{2}-(R / r)^{4}\left(r / r_{F}\right)^{4 \theta / d} r^{-2(z-1)} h(r)\right)\left(v^{2}-r^{-2(z-1)} h(r)\right)}}{r^{-(z-1)}} .
$$

In order to solve Schrödinger equation with some approximations, we divide the range $r_{F}<r<r_{H}$ in three regions $r \ll r_{H}, r \simeq r_{H}$, and $r_{t p} \ll r<r_{H}$.

Near Boundary $\left(r \ll r_{s}\right)$. In this region for $z=1$ and $z>1$ we have different limits as

$$
L=\left\{\begin{array}{l}
\frac{R^{2} r^{(2 \theta / d)-2}}{r_{F}^{2 \theta / d} \gamma} \\
\frac{R^{2} r^{(2 \theta / d)-1-z}}{r_{F}^{2 \theta / d}},
\end{array} \quad N= \begin{cases}\frac{R^{2} r^{(2 \theta / d)-2}}{r_{F}^{2 \theta / d}} & z=1, \\
\frac{R^{2} r^{(2 \theta / d)-2}}{r_{F}^{2 \theta / d}} & z>1 .\end{cases}\right.
$$

By using these relations the Schrödinger potential is given by $V_{s}$

$$
\simeq \begin{cases}-\gamma^{2} \omega^{2}+\frac{((\theta / d)-1)((\theta / d)-2)}{r^{2}} & z=1, \\ \frac{-\omega^{2}}{r^{-2(z-1)}} & \\ +\frac{((\theta / d)-(1 / 2)(z+1))((\theta / d)-(1 / 2)(z+3))}{r^{2}} & z>1 .\end{cases}
$$

We replace these potentials to relation (68); then the solution of Schrödinger equation will be

$$
\begin{gathered}
\phi=A_{1} \sqrt{r} J_{(3 d-2 \theta) / 2 d}(r \gamma \omega)+A_{2} \sqrt{r} Y_{(3 d-2 \theta) / 2 d}(r \gamma \omega), \quad z=1 \\
\phi=A_{1} \sqrt{r} J_{((z+2) d-2 \theta) / 2 z d}\left(\frac{r^{z} \omega}{z}\right) \\
+A_{2} \sqrt{r} Y_{((z+2) d-2 \theta) / 2 z d}\left(\frac{r^{z} \omega}{z}\right), \quad z>1 .
\end{gathered}
$$

Near Horizon $\left(r \simeq r_{s}\right)$. In this region for both $z=1$ and $z>1$ case we obtain

$$
L=\left(4 \pi T_{s}\right) \frac{R^{2} r_{s}^{(2 \theta / d)-2}}{r_{F}^{2 \theta / d}}\left(r_{s}-r\right) .
$$

If we implement the above relation and (64) for $N\left(r_{s}\right)$ in the Schrödinger potential one can arrive at

$$
V_{s} \simeq-\left(\widetilde{\omega}^{2}+\frac{1}{4}\right) \frac{1}{\left(r-r_{s}\right)^{2}}, \quad r \longrightarrow r_{s}
$$

where $\widetilde{\omega}=\omega / 4 \pi T_{s}$. The solution of Schrödinger equation (68) after substituting this potential in-falling boundary condition at the horizon is given by

$$
\phi_{h} \simeq C_{h}\left(r_{s}-r\right)^{-i \widetilde{\omega}+(1 / 2)} .
$$

WKB Region $\left(r_{t p}<r \ll r_{s}\right)$. This region is allowed classically and it covers almost all ranges as $r_{F}<r<r_{s}$. For large $\omega$ 's, the first term of (68) dominates and the potential becomes

$$
V_{s} \simeq-\frac{\omega^{2} G_{i i}^{2}}{R^{2}}, \quad r_{t p}<r \ll r_{s} .
$$

For a small region close to the boundary, including turning point $r_{t p}$, the approximation (76) breaks down. The turning point for large $\omega$ 's is found by solving the equation, $V_{s}(r)=0$,

$$
\begin{aligned}
& r_{t p} \\
& =\left\{\begin{array}{l}
\frac{\sqrt{((\theta / d)-1)((\theta / d)-2)}}{\gamma \omega} \\
z=1, \\
{\left[\frac{((\theta / d)-(1 / 2)(z+1))((\theta / d)-(1 / 2)(z+3))}{\omega^{2}}\right]^{1 / 2 z}} \\
z>1 .
\end{array}\right.
\end{aligned}
$$

The crucial fact is that, for large $\omega, r_{t p} \ll r_{s}$, the regions 1 and 3 overlap. On the other hand, also regions 2 and 3 overlap and are close to $r \simeq r_{s}$. Therefore, the solution in WKB region can be used to connect the near-boundary and near-horizon. By inserting the expression (76) in (68) two independent solutions to $-\phi^{\prime \prime}+V \phi=0$ in the region $V \ll 0$ are written as

$$
\begin{gathered}
\phi_{1}=\frac{1}{\sqrt{p}} \cos \int^{r} p, \quad \phi_{2}=\frac{1}{\sqrt{p}} \sin \int^{r} p, \\
p(r)=\sqrt{-V_{s}(r) .}
\end{gathered}
$$

Explicitly, the general solution has the following form:

$$
\begin{array}{r}
\phi_{w k b}=C_{1} \frac{\sqrt{L}}{G_{i i}} \cos \int^{r} \frac{\omega^{2} G_{i i}}{L}+C_{2} \frac{\sqrt{L}}{G_{i i}} \sin \int^{r} \frac{\omega^{2} G_{i i}}{L}, \\
r_{t p}<r \ll r_{s} .
\end{array}
$$

We should note that the solutions in three regions are applied for both transversal and longitudinal equations.

In the next step, we consider the cases that three regions overlap. As we mentioned before, regions 2 and 3 overlap close to the horizon. By expanding the solutions (79) for large $\omega$ 's near the horizon we have the following equation,

$$
\begin{aligned}
& \phi_{w k b} \\
& \simeq\left(4 \pi T_{s}\right)^{1 / 2}\left(r_{s}-r\right)^{1 / 2} \\
& \quad \times\left\{C_{1} \cos \left[v-\widetilde{\omega} \log \left(r_{s}-r\right)\right]+C_{2} \sin \left[v-\widetilde{\omega} \log \left(r_{s}-r\right)\right]\right\},
\end{aligned}
$$$$
r \longrightarrow r_{s} \text {, }
$$ 
where $v=\int_{0}^{r_{1}}\left(\omega^{2} G_{i i} / L\right)$. By comparing relations (75) and (80) we find that

$$
C_{1}=-i C_{2}=\frac{C_{h}}{\left(4 \pi T_{s}\right)^{1 / 2}} e^{-i v} .
$$

Next we consider the near-boundary region $r \ll r_{s}$. We know that, for the large $\omega$ 's, the UV region overlaps with the WKB region. For matching the UV solutions (72) for large $\omega$ 's, we need the following expansion for Bessel functions:

$$
\begin{array}{ll}
J_{\nu}(x) \simeq \sqrt{\frac{2}{\pi x}}\left[\cos \left(x-\frac{v \pi}{2}-\frac{\pi}{4}\right)+\cdots\right], \quad|x| \longrightarrow \infty, \\
Y_{\nu}(x) \simeq \sqrt{\frac{2}{\pi x}}\left[\sin \left(x-\frac{v \pi}{2}-\frac{\pi}{4}\right)+\cdots\right], & |x| \longrightarrow \infty .
\end{array}
$$

So, the large $\omega$ 's expansion for solutions (72) becomes

$$
\begin{aligned}
& \phi_{u v}= A_{1} \sqrt{\frac{2}{\pi \gamma \omega}}\left[\cos \left(\gamma \omega r-\frac{\pi}{2}\left(\frac{2 d-\theta}{d}\right)\right)\right] \\
&+A_{2} \sqrt{\frac{2}{\pi \gamma \omega}}\left[\sin \left(\gamma \omega r-\frac{\pi}{2}\left(\frac{2 d-\theta}{d}\right)\right)\right], \\
& z=1, \\
& \phi_{u v}= A_{1} \sqrt{\frac{2 z}{\pi \omega r^{z-1}}}\left[\cos \left(\frac{\omega r^{z}}{z}-\frac{\pi}{2}\left(\frac{(z+1) d-\theta}{z d}\right)\right)\right] \\
&+A_{2} \sqrt{\frac{2 z}{\pi \omega r^{z-1}}}\left[\sin \left(\frac{\omega r^{z}}{z}-\frac{\pi}{2}\left(\frac{(z+1) d-\theta}{z d}\right)\right)\right], \\
& z>1 .
\end{aligned}
$$

On the other hand for WKB solutions in the $r \ll r_{s}$ we have

$$
\begin{gathered}
\phi_{w k b} \simeq \frac{C_{1}}{\sqrt{\gamma}} \cos (\gamma \omega r)+\frac{C_{2}}{\sqrt{\gamma}} \sin (\gamma \omega r), \quad r \ll r_{s} \quad \text { for } z=1, \\
\phi_{w k b} \simeq \frac{C_{1}}{\sqrt{r^{z-1}}} \cos \left(\frac{\omega r^{z}}{z}\right)+\frac{C_{2}}{\sqrt{r^{z-1}}} \sin \left(\frac{\omega r^{z}}{z}\right), \quad r \ll r_{s}
\end{gathered}
$$

for $z>1$.

Comparing between (83), (85) for $z=1$ and (84), (85) for $z>1$ gives us the following equation:

$$
\begin{gathered}
C_{1}=A_{1} e^{-i(\pi / 2)((2 d-\theta) / d)} \sqrt{\frac{2}{\pi \omega}}, \quad A_{2}=i A_{1} \quad \text { for } z=1, \\
C_{1}=A_{1} e^{-i(\pi / 2)(((z+1) d-\theta) / z d)} \sqrt{\frac{2 z}{\pi \omega}}, \quad A_{2}=i A_{1} \quad \text { for } z>1 .
\end{gathered}
$$

Finally, all coefficients depend on determination of $A_{2}$. By imposing unit normalization of the function $\psi=(1 / \sqrt{\mathscr{L}}) \phi$ at $r=r_{b}$, that is, the point where the string is attached, one can find this coefficient.

Infinite Quark Mass. In this case the end-point of string is attached to the boundary $r_{b}=r_{F} \rightarrow 0$ and we normalize the wave-functions on this location. Then by imposing the $\psi\left(r_{F} \rightarrow 0, \omega\right)=1$ we have

$$
\begin{gathered}
A_{2}=\frac{-\pi R(\omega / 2)^{(3 d-2 \theta) / 2 d} \gamma^{1-(\theta / d)}}{r_{F}^{\theta / d} \Gamma[(3 d-2 \theta) / 2 d]}, \quad z=1, \\
A_{2}=\frac{-\pi R(\omega / 2 z)^{((z+2) d-2 \theta) / 2 z d}}{r_{F}^{\theta / d} \Gamma[((z+2) d-2 \theta) / 2 z d]}, \quad z>1 .
\end{gathered}
$$

Consequently, by using (86) and (87) with (81) we get the following result:

$$
\begin{gathered}
C_{h}=\frac{\sqrt{\pi}(\omega \gamma / 2)^{1-(\theta / d)}\left(4 \pi T_{s}\right)^{1 / 2} R}{r_{F}^{\theta / d} \Gamma[(3 d-2 \theta) / 2 d]} e^{i(v-(\pi / 2)((d-\theta) / d))}, \quad z=1, \\
C_{h}=\frac{\sqrt{\pi}(\omega / 2 z)^{(d-\theta) / z d}\left(4 \pi T_{s}\right)^{1 / 2} R}{r_{F}^{\theta / d} \Gamma[((z+2) d-2 \theta) / 2 z d]} e^{i(v-(\pi / 2)((d-\theta) / z d))}, \quad z>1 .
\end{gathered}
$$

Eventually from the above expressions we derive the coefficient $\psi_{h}$ as

$$
\begin{aligned}
\psi_{h}= & \frac{\sqrt{\pi}(\omega \gamma / 2)^{1-(\theta / d)}}{r_{s}^{(\theta / d)-1} \Gamma[(3 d-2 \theta) / 2 d]} e^{i(v-(\pi / 2)((d-\theta) / d))} \\
& \times\left\{\begin{array}{lll}
1 & \perp \\
\sqrt{\frac{G_{i i}\left(r_{s}\right)}{N\left(r_{s}\right)}} \quad \|, & z=1,
\end{array}\right. \\
\psi_{h}= & \frac{\sqrt{\pi}(\omega / 2 z)^{(d-\theta) / z d}}{r_{s}^{(\theta / d)-1} \Gamma[((z+2) d-2 \theta) / 2 z d]} e^{i(v-(\pi / 2)((d-\theta) / z d))} \\
& \times\left\{\begin{array}{lll}
1 & \perp \\
\sqrt{\frac{G_{i i}\left(r_{s}\right)}{N\left(r_{s}\right)}} & \|, & z>1 .
\end{array}\right.
\end{aligned}
$$

By inserting these expressions in (65) we get

$$
\begin{gathered}
\operatorname{Im} G_{R}^{\perp}=\frac{R^{2} \omega}{2 \alpha^{\prime}} \frac{(\omega \gamma / 2)^{2-(2 \theta / d)}}{r_{F}^{2(\theta / d)}[\Gamma((3 d-2 \theta) / 2 d)]^{2}}, \quad z=1, \\
\operatorname{Im} G_{R}^{\perp}=\frac{R^{2} \omega}{2 \alpha^{\prime}} \frac{(\omega / 2 z)^{2(d-\theta) / z d}}{r_{F}^{2(\theta / d)}[\Gamma(((z+2) d-2 \theta) / 2 z d)]^{2}}, \quad z>1 .
\end{gathered}
$$

The longitudinal component of retarded correlator can be found easily by relations (64) and (65). As mentioned before the imaginary part of retarded correlator is proportional to the conserved current. By using the above expression for imaginary part of retarded correlator and (67), we determine 
the spectral densities associated with the Langevin dynamics in the limit $\omega \gg 1 / r_{s}$ :

$$
\begin{gathered}
\rho_{\perp}(\omega) \simeq \gamma^{-2} \rho_{\|}(\omega), \quad z=1, \\
\rho_{\perp}(\omega) \simeq \rho_{\|}(\omega), \quad z>1 .
\end{gathered}
$$

So this result is interesting only for $z=1$ for the transversal and the longitudinal components of spectral density. In the region $z>1$, this situation approximately converts to the equality for components of spectral densities.

Finite Quark Mass. Here we are going to study the finite mass quark which is a similar work for an infinitely case. Now, also we use relations (72) but with the normalization condition at the cutoff $r_{b}=r_{q} \neq 0$. In this case one can obtain

$$
\begin{aligned}
A_{2}= & \sqrt{\frac{L\left(r_{q}\right)}{r_{q}}} \\
& \times\left[-i J_{(3 d-2 \theta) / 2 d}\left(r_{q} \gamma \omega\right)+Y_{(3 d-2 \theta) / 2 d}\left(r_{q} \gamma \omega\right)\right], \\
A_{2}= & \sqrt{\frac{L\left(r_{q}\right)}{r_{q}}} z=1, \\
& \times\left[-i J_{((z+2) d-2 \theta) / 2 z d}\left(\frac{r_{q}^{z} \omega}{z}\right)+Y_{((z+2) d-2 \theta) / 2 z d}\left(\frac{r_{q}^{z} \omega}{z}\right)\right],
\end{aligned}
$$

The other coefficients can be derived through the above relation as with the previous way for the infinite mass case.

\subsection{Langevin Diffusion Constants via the Retarded Correlator.} So far, we found the correlators and spectral densities which are required to establish the generalized Langevin equation. Now we want to find the diffusion coefficients from the information of last section for both $z=1$ and $z$ > 1 case. We consider a long-time limit which makes the generalized Langevin equation. This limit is expressed in the zero-frequency limit of Green's functions [27, 28, 53, 54]. Therefore, we investigate the zero-frequency limit of Green's functions which allow us to give the analytic results for the diffusion constants. The diffusion constant is defined in terms of the symmetric correlator $G_{\text {sym }}$ as

$$
\kappa=\lim _{\omega \rightarrow 0} G_{\text {sym }}=-2 T_{s} \lim _{\omega \rightarrow 0} \frac{G_{R}(\omega)}{\omega} .
$$

By going back to the definition of correlator (63), it seems that the evaluation of wave-function in the zero-frequency limit is necessary. For this purpose, we write the small-frequency limit for the horizon asymptotic of the $\psi_{R}$ in (45):

$$
\begin{aligned}
& \psi_{R}(r, \omega) \\
& \quad=\psi_{h}\left(r_{s}-r\right)^{-i\left(\omega / 4 \pi T_{s}\right)} \simeq\left(1-\frac{-i \omega}{4 \pi T_{s}} \log \left|r_{s}-r\right|+\cdots\right) .
\end{aligned}
$$

This solution reduces to the $\psi_{R}=\psi_{h}$ in the strict limit $\omega=0$. It matches with the boundary solution results $\psi_{h}=1$ for both transverse and longitudinal modes. This condition is applied consistently for finite and infinite massive quarks, since the radius value for boundary does not have effect in (63) for low frequency limit. Therefore, by using the explicit expressions (65) in (94) and $\psi_{h}=1$, we receive the following results:

$$
\begin{gathered}
\kappa_{\perp}=\frac{R^{2} r_{s}^{(2 \theta / d)-2}}{\pi \alpha^{\prime} r_{F}^{2 \theta / d}} T_{s}, \\
\kappa_{\|}=\frac{16 \pi R^{2} r_{s}^{(2 \theta / d)+2 z-4} T_{s}^{3}}{\alpha^{\prime} r_{F}^{2 \theta / d} h^{2}\left(r_{s}\right)\left(\left(h^{\prime}\left(r_{s}\right) / h\left(r_{s}\right)\right)+\left(-2(z-1) / r_{s}\right)\right)^{2}} .
\end{gathered}
$$

From the above expression for the diffusion constants, it is obvious that there is not any dependence on dynamical exponent $z$ for transversal component but for longitudinal component. The ratio between transversal and longitudinal component can be written as

$$
\frac{\kappa_{\|}}{\kappa_{\perp}}=\left[\frac{4 \pi r_{s}^{z} T_{s}}{2(1-z)+(2+d-z-\theta)\left(v^{2}-r_{s}^{-2(z-1)}\right)}\right]^{2} .
$$

For the special case $z=1$ and using the definition of $T_{s}$ in (18), we get

$$
\frac{\kappa_{\|}}{\kappa_{\perp}}=1+\frac{4(d-\theta)}{d(d+1-\theta)}\left[\frac{v^{2}}{1-v^{2}}\right] .
$$

For particular gauge/gravity dualities the inequality $\kappa_{\|}>$ $\kappa_{\perp}$ has been noticed to hold [56, 57]. In the absence of hyperscaling parameter $\theta$ one can check that this inequality is maintained, but, in the presence of $\theta$, it seems that we need the $\theta<d$ condition. We note that at condition NEC; the range $\theta>d$ is allowed. But, in range of $\theta$ we have some instabilities in the gravity side. For the range of $z>1$ the ratio between the transversal and longitudinal components is given by

$$
\frac{\kappa_{\|}}{\kappa_{\perp}}=1+\frac{4(d-\theta) v^{2}}{d\left[(d+z-\theta) r_{s}^{-2(z-1)}-(2+d-z-\theta) v^{2}\right]}
$$

It is obvious that the range of $\theta<d$ is also necessary for the case $z>1$. In this case there is also another condition, $r_{s}^{2(z-1)}<(d+z-\theta) /\left(v^{2}(d+2-z-\theta)\right)$, with $r_{s}$ defined in (11). In the special case $\theta=d$ the universal inequality $\kappa_{\|}>\kappa_{\perp}$ converts to the equality $\kappa_{\|}=\kappa_{\perp}$ for both $z=1$ and $z>1$. For this special $\theta$ in the last section, we found that the Hawking temperature and modified temperature are identical. 
The jet-quenching parameters can be defined in terms of the diffusion constants as $[27,28]$

$$
\hat{q}^{\perp}=\frac{2 \kappa^{\perp}}{v}, \quad \hat{q}^{\|}=\frac{\kappa^{\|}}{v} .
$$

Therefore we obtain

$$
\begin{aligned}
\hat{q}^{\perp} & =2 \frac{R^{2} r_{s}^{(2 \theta / d)-2}}{v \pi \alpha^{\prime} r_{F}^{2 \theta / d}} T_{s}, \\
\hat{q}^{\|} & =\frac{\kappa_{\perp}}{v}\left[\frac{4 \pi r_{s}^{z} T_{s}}{2(1-z)+(2+d-z-\theta)\left(v^{2}-r_{s}^{-2(z-1)}\right)}\right]^{2} .
\end{aligned}
$$

3.4. The Diffusion Constants via the Membrane Paradigm. This method allows us to achieve the diffusion constants directly from action (26); here we do not need to derive the wave-function as a pervious section, which is described in detail by [58]. By using this method for the following metric background,

$$
d s^{2}=a(r)^{2} d t^{2}+b(r)^{2} d r^{2}+c_{i}^{2}(r) d x_{i}^{2}
$$

we obtain the transversal and longitudinal diffusion constant as

$$
\begin{gathered}
\kappa_{\perp}=\frac{c_{i}^{2}\left(r_{s}\right)}{\pi \alpha^{\prime}} T_{s}, \\
\kappa_{\|}= \pm\left.\frac{16 \pi}{\alpha^{\prime}} \frac{\left|a^{2}\right| b^{2}}{c_{i}^{2}\left(a^{2} / c_{i}^{2}\right)^{\prime}\left|\left(a^{2} / c_{i}^{2}\right)^{\prime}\right|}\right|_{r=r_{s}} T_{s}^{3} .
\end{gathered}
$$

By inserting the component of metric background (2), we receive expected result (96) for diffusion constants. In the next section we will study $R$-charged black hole with hyperscaling violation.

\section{4. $R$-Charged Black Holes with Hyperscaling Violation}

Generally, the $R$-charged black holes have three independent charges and are static solutions of $N=2$ supergravity. The bosonic part of the effective gauged supersymmetric $N=$ 2 Lagrangian describes the coupling of vector multiples in supergravity [59] which is given by the following expression,

$$
\begin{aligned}
e^{-1} L= & \left(\frac{\Re}{2}-\frac{1}{2} g_{x y} \partial_{\mu} \phi^{x} \partial^{\mu} \phi^{y}-\frac{1}{4} a_{I J} F_{\mu \nu}^{I} F^{\mu \nu J}\right. \\
& \left.-g^{2} V+\frac{e^{-1}}{48} \epsilon^{\mu \nu \rho \sigma \lambda} C_{I J K} F_{\mu \nu}^{I} F_{\rho \sigma}^{J} A_{\lambda}^{K}\right)
\end{aligned}
$$

where $e=\sqrt{-g}$ is the determinant of vielbein, $\mathfrak{R}$ is the Ricci scalar, $g_{x y}$ is a metric on the scalar manifold, $a_{I J}$ is a kinetic gauge coupling of the field strength, $g$ is a constant gauge coupling, and $g_{x y}, a_{I J}$, and $V$ are functions of scalar field $\phi^{x}$. The variation of Lagrangian (104) with respect to $g_{x y}, \phi^{x}$, and $F_{\mu \nu}^{I}$ gives the field equations of motion. In [36] we found the deformed $R$-charged black-hole metric background with hyperscaling violation. In this section we use the form of $R$-charged black hole with hyperscaling violation in the flat space $k=0$. The metric background of this black hole is given by

$$
d s^{2}=\left(\frac{r}{r_{F}}\right)^{2(\theta / 3)} \frac{R^{2}}{r^{2}}\left[H^{1 / 3}\left(-h d t^{2}+d x_{i}^{2}\right)+H^{-2 / 3} h^{-1} d r^{2}\right],
$$

with

$$
\begin{aligned}
& H=\left(\frac{r}{r_{F}}\right)^{\theta}+Q_{1} r^{2}+Q_{2} r_{F}^{\theta} r^{4-\theta}+Q_{3} r_{F}^{2 \theta} r^{6-2 \theta}, \\
& h=1-\left(\frac{r}{R}\right)^{2}\left(\frac{r}{r_{0}}\right)^{2-\theta} H^{-1},
\end{aligned}
$$

where $Q_{1}=\left(q_{1}+q_{2}+q_{3}\right) / R^{2}, Q_{2}=\left(q_{1} q_{2}+q_{2} q_{3}+\right.$ $\left.q_{1} q_{3}\right) / R^{4}$, and $Q_{3}=q_{1} q_{2} q_{3} / R^{6}$. Notice that, in the definition of metric background (105), we use the radial coordinate transformation $r \rightarrow\left(R^{2} / r\right)$. We introduce parameter $r_{h}$ as the location of horizon in the $r$ coordinate such that it is the largest root of $h=0$. Now, by using the above information, we are ready to proceed as pervious section.

4.1. Trailing String and Drag Force. In order to study the stochastic motion of the quark in a plasma on the boundary of $R$-charged black hole with hyperscaling violation, we repeat the process of Section 2 in this section. The trailing string corresponding to a quark moving on the boundary of $R$-charged black hole with a constant velocity $v$-through parameterization (6) - is characterized by the following induced worldsheet metric:

$$
\begin{aligned}
g_{a b}= & \left(\frac{R}{r}\right)^{2}\left(\frac{r}{r_{F}}\right)^{2 \theta / d} \\
& \times H(r)^{1 / 3}\left(\begin{array}{cc}
v^{2}-h(r) & v \xi^{\prime}(r) \\
v \xi^{\prime}(r) & (h(r) H(r))^{-1}+\xi^{\prime 2}
\end{array}\right) .
\end{aligned}
$$

Constructing the Nambu-Goto action we find the momentum $\pi_{\xi}$ flowing from the bulk to the horizon which is equal to the drag force:

$$
F_{\text {drag }}=\pi_{\xi}=\frac{v R^{2} H^{2 / 3}\left(r_{s}\right)}{2 \pi \alpha^{\prime} r_{F}^{2}}
$$

The stretched horizon $r_{s}$ is defined through the relation $h\left(r_{s}\right)=v^{2}$ and this expression reduces to solve the following equation,

$$
\left(1-v^{2}\right) r_{s}^{\theta}\left(1+a x+b x^{2}+c x^{3}\right)=0, \quad \text { with } x=r_{s}^{2-\theta} \text {, }
$$


where $a=Q_{1} r_{F}^{\theta}, b=Q_{2} r_{F}^{2 \theta}+\left(r_{F}^{\theta} / R^{2} r_{0}^{2-\theta}\left(1-v^{2}\right)\right)$, and $c=$ $Q_{3} r_{F}^{3 \theta}$. The stretched horizon for $\theta \neq 2$ in the terms of $a, b$, and $c$ is given by

$$
x=\frac{1}{3}\left[\frac{B}{2 c}-2 \frac{\left(3 a c-b^{2}\right)}{c B}-\frac{b}{c}\right]
$$

with $B=\left[12 \sqrt{3} \sqrt{4 a^{3} c-a^{2} b^{2}-18 a b c+4 b^{3}+27 c^{2}} c\right.$

$$
\left.+36 a b c-8 b^{3}-108 c^{2}\right]^{1 / 3} \text {. }
$$

For the special case $\theta=2$, in order to receive $r_{s} \neq 0$, there must be the condition $1+a+b+c=0$. If we diagonalize worldsheet induced metric (107), then the modified temperature is obtained as

$$
\begin{aligned}
T_{s}^{2}= & \frac{h^{\prime}\left(r_{s}\right) h\left(r_{s}\right) H\left(r_{s}\right)}{16 \pi^{2}} \\
& \times\left[\frac{4((\theta / 3)-1)}{r_{s}}+\frac{h^{\prime}\left(r_{s}\right)}{h\left(r_{s}\right)}+\frac{2}{3} \frac{H^{\prime}\left(r_{s}\right)}{H\left(r_{s}\right)}\right] .
\end{aligned}
$$

By calculating $h^{\prime}\left(r_{s}\right)$ in detail one can obtain that for $\theta=$ $3-1=2$ this expression becomes zero and hence the temperature. For this value of $\theta$, we obtained in [36] that the temperature and total particle number got zero. Therefore for $\theta=2$ the Hawking temperature and the modified temperature both become equal to zero.

4.2. Fluctuations of Trailing String. In order to investigate the fluctuations of trailing string we utilize quadratic NambuGoto action (26) with

$$
\begin{gathered}
N(r)=\frac{h(r)(R / r)^{4}\left(r / r_{F}\right)^{4 \theta / d} H(r)^{2 / 3}-C^{2}}{(R / r)^{2}\left(r / r_{F}\right)^{2 \theta / d} H(r)^{1 / 3}\left(h(r)-v^{2}\right)}, \\
G_{i i}=\left(\frac{R}{r}\right)^{4}\left(\frac{r}{r_{F}}\right)^{4 \theta / d} H(r)^{1 / 3} .
\end{gathered}
$$

Therefore, by using the above expressions equations of motion (28) become

$$
\begin{aligned}
& \partial_{r}\left[\sqrt{\left.H(r)\left(C^{2}-\left(\frac{R}{r}\right)^{4}\left(\frac{r}{r_{F}}\right)^{4 \theta / d} H(r)^{2 / 3} h(r)\right)\left(v^{2}-h(r)\right) \partial_{r} \delta X^{\perp}\right]}\right. \\
& +\frac{\omega^{2}(R / r)^{4}\left(r / r_{F}\right)^{4 \theta / d}}{\sqrt{H(r)\left(C^{2}-(R / r)^{4}\left(r / r_{F}\right)^{4 \theta / d} H(r)^{2 / 3} h(r)\right)\left(v^{2}-h(r)\right)}} \delta X^{\perp}=0, \\
& \partial_{r}\left[\frac{\left(C^{2}-(R / r)^{4}\left(r / r_{F}\right)^{4 \theta / d} h(r)\right) H(r)^{3 / 2}}{\left(v^{2}-h(r)\right)^{1 / 2} H(r)^{1 / 6}} \partial_{r} \delta X^{\|}\right]+\frac{\omega^{2}\left(C^{2}-(R / r)^{4}\left(r / r_{F}\right)^{4 \theta / d} H(r)^{2 / 3} h(r)\right)^{1 / 2}}{\left(v^{2}-h(r)\right)^{3 / 2} H(r)^{1 / 2}} \delta X^{\|}=0
\end{aligned}
$$

At the world-sheet horizon $r \rightarrow r_{s}$ both of the above equations reduce to (33) with $T_{s}$ defined in (111). Therefore, the solution to this equation is similar to what is obtained in relation (34). Near the boundary limit, $r \rightarrow r_{F}$, of (113), we receive relation (35) with $d=3$, since in this limit $h(r)$ and $H(r)$ have the following behavior:

$$
\lim _{r \rightarrow r_{F} \rightarrow 0} H(r)=1, \quad \lim _{r \rightarrow r_{F} \rightarrow 0} h(r)=1 .
$$

So the solutions to the equations of motion near the boundary are similar as before, that is, relation (36) with $d=3$.

4.3. Momentum Correlator of Trailing String. The classical solutions that we obtained for a trailing string in blackhole background (105) are implemented in computing the Langevin correlators. As we discussed before, these correlators involve two parts: real and imaginary.
4.3.1. Real Part of Retarded Correlator. The real part of the correlators from fluctuation modes (36) is obtained through relation (31) as

$$
\begin{aligned}
\operatorname{Re} G_{R}^{\perp} \simeq & \gamma^{-2} \operatorname{Re} G_{R}^{\|} \simeq \frac{\gamma \omega^{2}}{2 \pi \alpha^{\prime}(1-(2 \theta / 3)) r_{F}} \\
& \times\left[1+\frac{\gamma^{2} \omega^{2} r_{F}^{2}}{2(1-(2 \theta / 3))}+O\left(r_{F}^{4}\right)\right]
\end{aligned}
$$

As this relation demonstrates, in the limit of $r_{F} \rightarrow 0$, there are some divergencies of the order of $\left(1 / r_{F}\right)$. These divergencies can be canceled in a similar way to Section 2 by the identification of $\Delta M\left(r_{F}\right)=-\left(R^{2} /\left(2 \pi \alpha^{\prime}(1-(2 \theta / 3)) r_{F}\right)\right)$ in boundary counterterm (55).

4.3.2. Imaginary Part of Retarded Correlator. For computing the imaginary part of retarded correlator, we require relation 
(63). The expressions $g_{\perp}^{r r}$ and $g_{\|}^{r r}$ in relation (30) reduce to the following for metric background (105):

$$
\begin{array}{r}
g_{\perp}^{r r} \simeq 4 \pi T_{s}\left(r_{s}-r\right) G_{i i}\left(r_{s}\right), \quad g_{\|}^{r r} \simeq 4 \pi T_{s}\left(r_{s}-r\right) N\left(r_{s}\right), \\
r \longrightarrow r_{s},
\end{array}
$$

with $N\left(r_{s}\right)=\frac{16 \pi^{2} R^{2} T_{s}^{2}}{r_{F}^{2 \theta / d} H^{2 / 3}\left(r_{s}\right) h^{\prime 2}\left(r_{s}\right)} r_{s}^{(2 \theta / d)-2}$

By inserting the above expressions in (63) and using solution (44) for $\psi$ we write

$$
\operatorname{Im} G_{R}^{\perp}=-\frac{G_{i i}\left(r_{s}\right)\left|\psi_{h}^{\perp}\right|^{2}}{2 \pi \alpha^{\prime}} \omega, \quad \operatorname{Im} G_{R}^{\|}=-\frac{N\left(r_{s}\right)\left|\psi_{h}^{\|}\right|^{2}}{2 \pi \alpha^{\prime}} \omega .
$$

The spectral densities forming these correlators can be found via relation (67). To obtain the high-frequency behavior of the Langevin correlators, we will implement the WKB method in the next subsection.

4.4. The $W K B$ Approximation at Large Frequency. In this section we implement the WKB method to obtain the highfrequency behavior of spectral densities. Everything is the same as Section 2, except that the function $L$ has the following definition in Schrödinger potential (68):

$L$

$$
=\sqrt{H(r)\left(C^{2}-\left(\frac{R}{r}\right)^{4}\left(\frac{r}{r_{F}}\right)^{4 \theta / d} h(r) H^{2 / 3}(r)\right)\left(v^{2}-h(r)\right)} .
$$

As before, we divide the range of $r_{F}<r<r_{H}$ in three regions and derive the solutions to the Schrödinger equations.

Near Boundary $\left(r \ll r_{s}\right)$. In this region with

$$
L=\frac{R^{2} r^{(2 \theta / 3)-2}}{r_{F}^{2 \theta / 3} \gamma}, \quad N=\frac{R^{2} r^{(2 \theta / 3)-2}}{r_{F}^{2 \theta / 3}}
$$

and so, the Schrödinger potential,

$$
V_{s} \simeq-\gamma^{2} \omega^{2}+\frac{((\theta / 3)-1)((\theta / 3)-2)}{r^{2}}
$$

we receive the following solutions:

$$
\phi=A_{1} \sqrt{r} J_{(3 / 2)-(\theta / 3)}(r \gamma \omega)+A_{2} \sqrt{r} Y_{(3 / 2)-(\theta / 3)}(r \gamma \omega) \text {. }
$$

Near Horizon $\left(r \simeq r_{s}\right)$. In this region, one can obtain the following for $L$ function:

$$
L=\left(4 \pi T_{s}\right) \frac{R^{2} r_{s}^{(2 \theta / 2)-2}}{r_{F}^{2 \theta / 3}} H^{1 / 3}\left(r_{s}\right)\left(r_{s}-r\right)
$$

If we implement the above relation and relation (117) for $N\left(r_{s}\right)$ in the Schrödinger potential; then we get

$$
V_{s} \simeq-\left(\widetilde{\omega}^{2}+\frac{1}{4}\right) \frac{1}{\left(r-r_{s}\right)^{2}} \quad r \longrightarrow r_{s},
$$

where $\widetilde{\omega}=\omega / 4 \pi T_{s}$. The solution to Schrödinger equation (68) after substituting this potential is identical to what is found in solution (75).

WKB Region $\left(r_{t p}<r \ll r_{s}\right)$. In this region, for large $\omega$ 's, the first term in (68) dominates and the Schrödinger potential becomes

$$
V_{s} \simeq-\frac{\omega^{2} G_{i i}^{2}}{R^{2}}, \quad r_{t p}<r \ll r_{s}
$$

The turning point for large $\omega$ is found as

$$
r_{t p}=\frac{\sqrt{((\theta / 3)-1)((\theta / 3)-2)}}{\gamma \omega} .
$$

We should note that the solutions in three regions are applied for both transversal and longitudinal equations.

Now, with the above information, we are able to find the coefficients by considering the limits that three regions overlap. Eventually, by repeating the process followed in Section 2, we obtain the following expression for $\psi_{h}$ for the infinite massive quark case:

$$
\begin{aligned}
\psi_{h}= & \frac{\sqrt{\pi}(\omega \gamma / 2)^{1-(\theta / 3)}}{r_{s}^{(\theta / 3)-1} \Gamma[(3 / 2)-(\theta / 3)]} e^{i(v-(\pi / 2)((3-\theta) / 3))} \\
& \times \begin{cases}1 & \perp \\
\sqrt{\frac{G_{i i}\left(r_{s}\right)}{N\left(r_{s}\right)}} & \|\end{cases}
\end{aligned}
$$

and then by inserting the above expressions in (63) we attain

$$
\operatorname{Im} G_{R}^{\perp}=\frac{R^{2} \omega}{2 \alpha^{\prime}} \frac{(\omega \gamma / 2)^{2-(2 \theta / 3)}}{r_{F}^{2(\theta / 3)}[\Gamma((3 / 2)-(\theta / 3))]^{2}} .
$$

The longitudinal component can be found in the same way and by using relation (65). The finite mass case is obtained through the way described in Section 2.

4.5. Langevin Diffusion Constants. The diffusion coefficients can be found either from the direct evaluation of the correlators or from using the membrane paradigm. Both of these methods give rise to the following identical results for diffusion constants:

$$
\begin{gathered}
\kappa_{\perp}=\frac{R^{2} H^{1 / 3}\left(r_{s}\right) r_{s}^{(2 \theta / 3)-2}}{\pi \alpha^{\prime} r_{F}^{2 \theta / 3}} T_{s}, \\
\kappa_{\|}=\frac{16 \pi R^{2} r_{s}^{(2 \theta / 3)-2} T_{s}^{3}}{\alpha^{\prime} r_{F}^{2 \theta / 3} H^{2 / 3}\left(r_{s}\right) h^{\prime 2}\left(r_{s}\right)} .
\end{gathered}
$$


There is a problem in computing the longitudinal component for the special case $\theta=2$. In this case both $H\left(r_{s}\right)$ and $h^{\prime}\left(r_{s}\right)$ tend to zero, so the longitudinal diffusion constant tends to the infinity. It seems that this value for $\theta$ is unacceptable. By studying [36], we perceive that the null energy condition imposes some condition on $\theta$ as

$$
\theta \leq 0.365, \quad \theta \geq 1.315 .
$$

In a similar way to the previous work and because we see some instabilities in the range of $\theta \geq 1.315$, we choose the range of $\theta \leq 1.315$. This case is in analogy with the case $\theta<d$ in the previous section.

The ratio between longitudinal and transversal components of diffusion constants is

$$
\frac{\kappa_{\|}}{\kappa_{\perp}}=1+2 \frac{h\left(r_{s}\right)}{h^{\prime}\left(r_{s}\right)}\left[\frac{(2 \theta / 3)-2}{r_{s}}+\frac{H^{\prime}\left(r_{s}\right)}{\left(r_{s}\right)}\right] .
$$

We expect the universal inequality $\kappa_{\|}>\kappa_{\perp}$ to be held. Therefore, in order to keep the inequality for the following relation,

$$
\begin{aligned}
\frac{\kappa_{\|}}{\kappa_{\perp}}=1 & +2 \frac{v^{2}}{\left(1-v^{2}\right)} \\
& \times\left[\frac{\left(r_{s} / r_{F}\right)^{\theta}+\left(2 Q_{1} r_{s}^{2} / 3\right)+\left(Q_{2} r_{F}^{\theta} r_{s}^{4-\theta} / 3\right)}{2\left(r_{s} / r_{F}\right)^{\theta}+Q_{1} r_{s}^{2}-Q_{3} r_{F}^{2 \theta} r_{s}^{6-2 \theta}}\right],
\end{aligned}
$$

with $Q_{i}>0$, the condition $2+a x>c x^{3}$ must be satisfied. For $Q_{i}<0$, both of the numerator and denominator on the righthand side of relation (131) can be negative or positive, where each of these situations puts some conditions on $Q_{i}$ and $r_{s}$. Since, due to relation (110), the $r_{s}$ is related to $Q_{i}, r_{F}$, and $v^{2}$, to hold the inequality, there should be some conditions on $Q_{i}, r_{F}$, and $v^{2}$. The jet-quenching parameters can be obtained easily from relation (100) as in the previous section.

\section{Summary}

In this paper, we used AdS/CFT correspondence and studied the stochastic motion of an external quark in a plasma. It corresponds to fundamental string whose end-point lies in the UV region of a bulk black-hole background and is forced to move with velocity $v$. By using the Nambu-Goto action, we obtained the equations of motion for this string in the planar black holes with hyperscaling violation background. The solution of the corresponding equations of motion leads us to finding the classical profile of the trailing string. Next, we considered small fluctuations around the classical string profile. These fluctuations satisfied the second-order radial equations and are related to the associated thermal correlators. We achieved the modified temperature $T_{s}$; it was felt by string fluctuations. In order to have a positive temperature, we got a constraint on $\theta$ for the case of $z=$ 1. We derived the drag force on the quark in the presence of dynamical exponents $z=1$ and $z>1$. Here, we found that in the ultrarelativistic limit this force becomes zero for the case $z=1$, while for the range of $z>1$ it is not so. We computed holographically the full Langevin correlators and the associated spectral densities, including real and imaginary parts of correlators for large- and smallfrequency limits. In the large-frequency regime, the spectral densities are obtained via the modified WKB method. We have shown that only for the case $z=1$ the ratio between transversal and longitudinal components of spectral density depends on the velocity of quarks. For the range of $z>$ 1 this relation tends to a constant with the value of 1 . We investigated the large-time limit of the fluctuations and for the constant diffusion we need small-frequency modes to obtain the diffusion. In order to hold inequality $\kappa_{\|} \geq \kappa_{\perp}$, we have shown that the range of $\theta \leq d$ is an acceptable region for $\theta$. This region is in agreement with what is found in literature. We note that the region $\theta>d$ leads to some instabilities on the gravity side, so for this region we cannot have the physically consistent theories. For the special case $\theta=d$ the inequality $\kappa_{\|} \geq \kappa_{\perp}$ converts to the equality $\kappa_{\|}=\kappa_{\perp}$ for both $z=1$ and $z>1$ cases. In this case we found that the Hawking temperature and the modified temperature become equal; however the world-sheet horizon and the black-hole horizon are different.

We repeated all of the above procedures for the $R$-charged black hole with hyperscaling violation. For this black hole we found that the range of $\theta \leq 1.315$ can provide a plausible region for $\theta$ which is in agreement with our results in [36]. We also realized that the universal inequality of diffusion constants can be confirmed by some constraints on the worldsheet horizon and charges.

As we indicated before, we obtained the real part and imaginary part of retarded correlator for both planer and $R$ charged black-hole with hyperscaling violation. For real part, we encountered some divergencies in the limit $r \rightarrow r_{F}$. In that case we introduced a boundary counterterm action for the case $z=1$ in planar black hole and $R$-charged black hole with hyperscaling violation. For the case of $z>1$ in planar black hole with hyperscaling violation, the boundary counterterm with the last definition cannot help us to remove the divergencies. It seems that a new definition of boundary counterterm is needed to overcome this problem. We would like to investigate this problem in future works. We are also interested to study different black holes with hyperscaling violation and investigate the Langevin diffusion process.

\section{Conflict of Interests}

The authors declare that there is no conflict of interests regarding the publication of this paper.

\section{References}

[1] S. A. Bass, M. Gyulassy, H. Stöcker, and W. Greiner, "Signatures of quark-gluon plasma formation in high energy heavy-ion collisions: a critical review," Journal of Physics G: Nuclear and Particle Physics, vol. 25, no. 3, pp. R1-R57, 1999.

[2] V. M. Emelayanov, Y. P. Nikitin, and A. V. Vanyashin, "Introduction to Quark-Gluon plasma," Fortschritte der Physik, vol. 88, no. 1, pp. 1-34, 1990. 
[3] R. A. Janik, "The dynamics of quark-gluon plasma and AdS/CFT," Lecture Notes in Physics, vol. 828, pp. 147-181, 2011.

[4] J. Casalderrey-Solana, H. Liu, D. Mateos, K. Rajagopal, and U. A. Wiedemann, "Gauge/string duality, hot QCD and heavy ion collisions," http://arxiv.org/abs/1101.0618.

[5] J. Maldacena, "The large $N$ limit of superconformal field theories and supergravity," Advances in Theoretical and Mathematical Physics, vol. 2, no. 2, pp. 231-252, 1998.

[6] J. L. Petersen, "Introduction to the Maldacena conjecture on AdS/CFT," International Journal of Modern Physics A, vol. 14, no. 23, pp. 3597-3672, 1999.

[7] S. S. Gubser, I. R. Klebanov, and A. M. Polyakov, "Gauge theory correlators from non-critical string theory," Physics Letters B, vol. 428, no. 1-2, pp. 105-114, 1998.

[8] E. Witten, "Anti de Sitter space and holography," Advances in Theoretical and Mathematical Physics, vol. 2, no. 2, pp. 253-291, 1998.

[9] L. F. Cugliandolo, J. Kurchan, and L. Peliti, "Energy flow, partial equilibration, and effective temperatures in systems with slow dynamics," Physical Review E, vol. 55, no. 4, pp. 3898-3914, 1997.

[10] J. Casalderrey-Solana and D. Teaney, "Transverse momentum broadening of a fast quark in a $N=4$ Yang Mills plasma," Journal of High Energy Physics, vol. 2007, no. 4, 2007.

[11] D. T. Son and D. Teaney, "Thermal noise and stochastic strings in AdS/CFT," Journal of High Energy Physics A, vol. 2009, no. 7, article 021, 2009.

[12] G. C. Giecold, E. Iancu, and A. H. Mueller, "Stochastic trailing string and Langevin dynamics from AdS/CFT," Journal of High Energy Physics A, vol. 2009, no. 7, article 33, 2009.

[13] S. Nakamura and H. Ooguri, "Out of equilibrium temperature from holography," Physical Review D, vol. 88, Article ID 126003, 2013.

[14] G. E. Uhlenbeck and L. S. Ornstein, "On the theory of the Brownian motion," Physical Review, vol. 36, no. 5, pp. 823-841, 1930.

[15] P. Langevin, "On the theory of brownian motion," Comptes Rendus de l'Académie des Sciences, vol. 146, pp. 530-533, 1908.

[16] J. Boer, V. E. Hubeny, M. Rangamania, and M. Shigemori, "Brownian motion in AdS /CFT," Journal of High Energy Physics, vol. 2009, no. 07, p. 094, 2009.

[17] A. N. Atmaja, J. de Boer, and M. Shigemori, "Holographic Brownian motion and time scales in strongly coupled plasmas," Nuclear Physics B, vol. 880, pp. 23-75, 2014.

[18] A. N. Atmaja, "Holograghic Brownian motion in two dimensional rotating black hole," Journal of High Energy Physics, vol. 2013, no. 4, article 021, 2013.

[19] W. Fischler, J. F. Pedraza, and W. Tangarife Garcia, "Holographic brownian motion in magnetic environments," Journal of High Energy Physics, vol. 2012, no. 12, article 2, 2012.

[20] L. Huijse, S. Sachdev, and B. Swingle, "Hidden Fermi surfaces in compressible states of gauge-gravity duality," Physical Review B: Condensed Matter and Materials Physics, vol. 85, no. 3, Article ID 035121, 2012.

[21] C. P. Herzog and D. T. Son, "Schwinger-Keldysh propagators from AdS/CFT correspondence," Journal of High Energy Physics, vol. 2003, article 046, 2003.

[22] S. S. Gubser, "Momentum fluctuations of heavy quarks in the gauge-string duality," Nuclear Physics B, vol. 790, no. 1-2, pp. 175-199, 2008.
[23] F. Debbasch, K. Mallick, and J. P. Rivet, "Relativistic OrnsteinUhlenbeck process," Journal of Statistical Physics, vol. 88, no. 34, pp. 945-966, 1997.

[24] F. Debbasch and J. P. Rivet, "A diffusion equation from the relativistic Ornstein-Uhlenbeck process," Journal of Statistical Physics, vol. 90, no. 5-6, pp. 1179-1199, 1998.

[25] C. Chevalier and F. Debbasch, "Relativistic diffusions: a unifying approach," Journal of Mathematical Physics, vol. 49, Article ID 043303, 2008.

[26] J. Dunkel and P. Hänggi, "Relativistic Brownian motion," Physics Reports A: Review Section of Physics Letters, vol. 471, no. 1, pp. $1-73,2009$.

[27] D. Giataganas and H. Soltanpanahi, "Universal properties of the Langevin diffusion coefficients," Physical Review D-Particles, Fields, Gravitation and Cosmology, vol. 89, no. 2, Article ID 026011, 2014.

[28] U. Gursoy, E. Kiritsis, L. Mazzanti, and F. Nitti, "Langevin diffusion of heavy quarks in non-conformal holographic backgrounds," Journal of High Energy Physics, vol. 1012, article 088, 2010.

[29] J. Sadeghi, F. Pourasadollah, and H. Vaez, "Holograghic brownian motion in three dimensional Gödel black hole," Advances in High Energy Physics, vol. 2014, Article ID 762151, 9 pages, 2014.

[30] S. Ryu and T. Takayanagi, "Aspects of holographic entanglement entropy," Journal of High Energy Physics A, vol. 2006, no. 8, artcile 045, 2006.

[31] J. Sadeghi, B. Pourhassan, and F. Pourasadollah, "Holograghic Brownian motion in $2+1$ dimensional hairy black holes," The European Physical Journal C, vol. 74, article 2793, 2014.

[32] N. Ogawa, T. Takayanagi, and T. Ugajin, "Holographic Fermi surfaces and entanglement entropy," Journal of High Energy Physics, vol. 2012, article 125, 2012.

[33] M. Edalati, J. F. Pedraza, and W. T. Garcia, "Quantum fluctuations in holographic theories with hyperscaling violation," Physical Review D, vol. 87, no. 4, Article ID 046001, 2013.

[34] X. Dong, S. Harrison, S. Kachru, G. Torroba, and H. Wang, "Aspects of holography for theories with hyperscaling violation," Journal of High Energy Physics, vol. 2012, p. 41, 2012.

[35] B. S. Kim, "Schrödinger holography with and without hyperscaling violation," Journal of High Energy Physics, vol. 2012, no. 6, article 116, 2012.

[36] J. Sadeghi, B. Pourhassan, and F. Pourasadollah, "Thermodynamics of Schrödinger black holes with hyperscaling violation," Physics Letters B, vol. 720, no. 1-3, pp. 244-249, 2013.

[37] J. Sadeghi, B. Pourhassan, and A. Asadi, "Thermodynamics of string black hole with hyperscaling violation," The European Physical Journal C, vol. 74, article 2680, 2014.

[38] M. Cadoni and S. Mignemi, "Phase transition and hyperscaling violation for scalar black branes," Journal of High Energy Physics, vol. 2012, no. 6, article 056, 2012.

[39] M. Alishahiha and H. Yavartanoo, "On holography with hyperscaling violation," Journal of High Energy Physics, vol. 2012, article 34, 2012.

[40] M. Alishahiha, E. Ó. Colgáin, and H. Yavartanoo, "Charged black branes with hyperscaling violating factor," Journal of High Energy Physics, vol. 2012, no. 11, article 137, 2012.

[41] C. Charmousis, B. Gouteraux, B. S. Kim, E. Kiritsis, and R. Meyer, "Effective holographic theories for low-temperature condensed matter systems," Journal of High Energy Physics, vol. 2010, no. 11, article 151, 2010. 
[42] B. Gouteraux and E. Kiritsis, "Generalized holographic quantum criticality at finite density," Journal of High Energy Physics, vol. 2011, article 36, 2011.

[43] S. Ryu and T. Takayanagi, "Holographic derivation of entanglement entropy from the anti-de Sitter space/conformal field theory correspondence," Physical Review Letters, vol. 96, no. 18, Article ID 181602, 4 pages, 2006.

[44] P. Bueno, W. Chemissany, P. Meessen, T. Ortin, and C. S. Shahbazi, "Lifshitz -like solutions with hyperscaling violation in ungauged supergravity," http://arxiv.org/abs/1209.4047.

[45] S. Kachru, X. Liu, and M. Mulligan, "Gravity duals of Lifshitzlike fixed points," Physical Review D: Particles, Fields, Gravitation, and Cosmology, vol. 78, no. 10, Article ID 106005, 8 pages, 2008.

[46] M. Taylor, "Non-relativistic holography," http://arxiv.org/abs/ 0812.0530.

[47] S. S. Gubser, "Drag force in AdS/CFT" Physical Review D, vol. 74, no. 12, Article ID 126005, 2006.

[48] C. Charmousis, B. Gouteraux, B. S. Kim, E. Kiritsis, and R. Meyer, "Effective holographic theories for low-temperature condensed matter systems," Journal of High Energy Physics, vol. 1011, article 151, 2010.

[49] U. Gürsoy, E. Kiritsis, G. Michalogiorgakis, and F. Nitti, “Thermal transport and drag force in improved holographic QCD," Journal of High Energy Physics, vol. 2009, no. 12, article 056, 2009.

[50] D. T. Son and A. O. Starinets, "Minkowski-space correlators in AdS/CFT correspondence: recipe and applications," Journal of High Energy Physics, vol. 2002, no. 09, article 042, 2002.

[51] C. Hoyos and P. Koroteev, "On the null energy condition and causality in Lifshitz holography," Physical Review D, vol. 82, Article ID 084002, 2010.

[52] C. Hoyos and P. Koroteev, "On the null energy condition and causality in lifshitz holography," Physical Review D, vol. 82, Article ID 109905, 2010.

[53] J. Casalderrey-Solana and D. Teaney, "Transverse momentum broadening of a fast quark in a $\mathcal{N}=4$ Yang Mills plasma," Journal of High Energy Physics, vol. 2007, no. 4, article 039, 2007.

[54] G. C. Giecold, E. Iancu, and A. H. Mueller, "Stochastic trailing string and langevin dynamics from AdS /CFT," Journal of High Energy Physics, vol. 2009, article 33, 2009.

[55] D. Teaney, "Finite temperature spectral densities of momentum and $R$-charge correlators in $\mathcal{N}=4$ Yang Mills theory," Physical Review D, vol. 74, no. 4, Article ID 045025, 11 pages, 2006.

[56] S. Nakamura and H. Ooguri, "Out of equilibrium temperature from holography," Physical Review D, vol. 88, no. 12, Article ID 126003, 2013.

[57] U. Gursoy, E. Kiritsis, L. Mazzanti, and F. Nitti, "Langevin diffusion of heavy quarks in non-conformal holographic backgrounds," Journal of High Energy Physics, vol. 1012, article 088, 2010.

[58] N. Iqbal and H. Liu, "Universality of the hydrodynamic limit in AdS/CFT and the membrane paradigm," Physical Review D, vol. 79, Article ID 025023, 2009.

[59] K. Behrndt, M. Cvetič, and W. A. Sabra, "Non-extreme black holes of five-dimensional $N=2$ AdS supergravity," Nuclear Physics B, vol. 553, no. 1-2, pp. 317-332, 1999. 

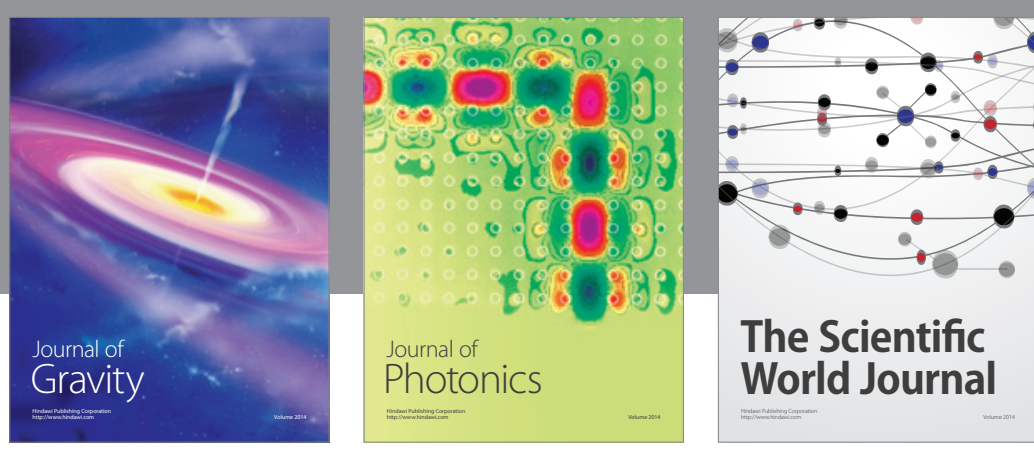

The Scientific World Journal
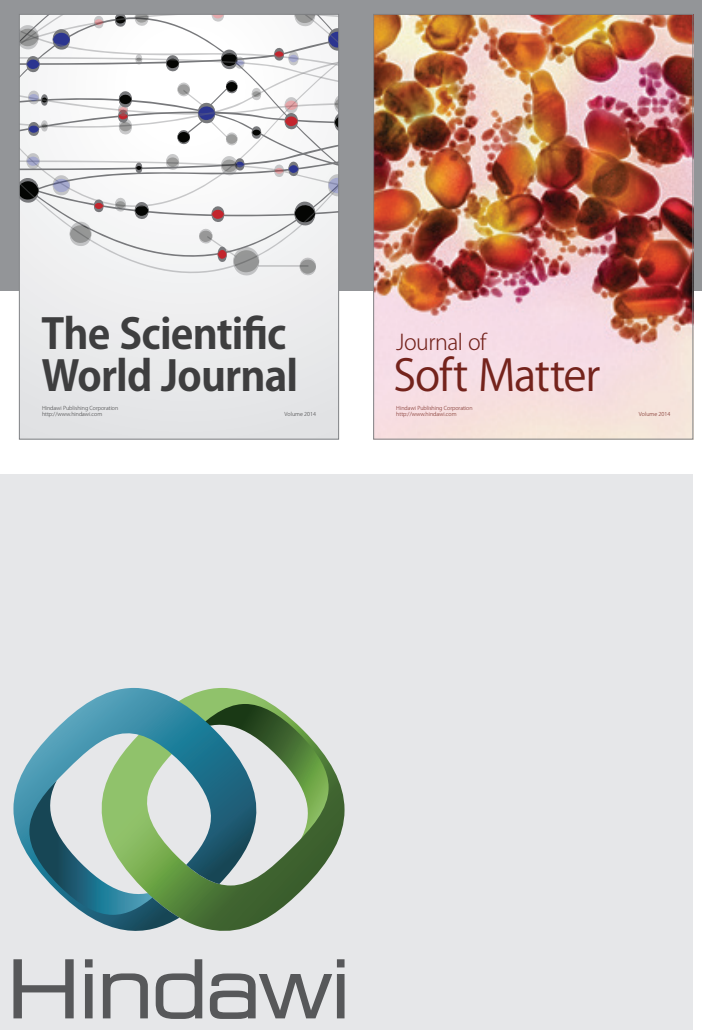

Submit your manuscripts at

http://www.hindawi.com

nternational Journal of

Statistical Mechanics
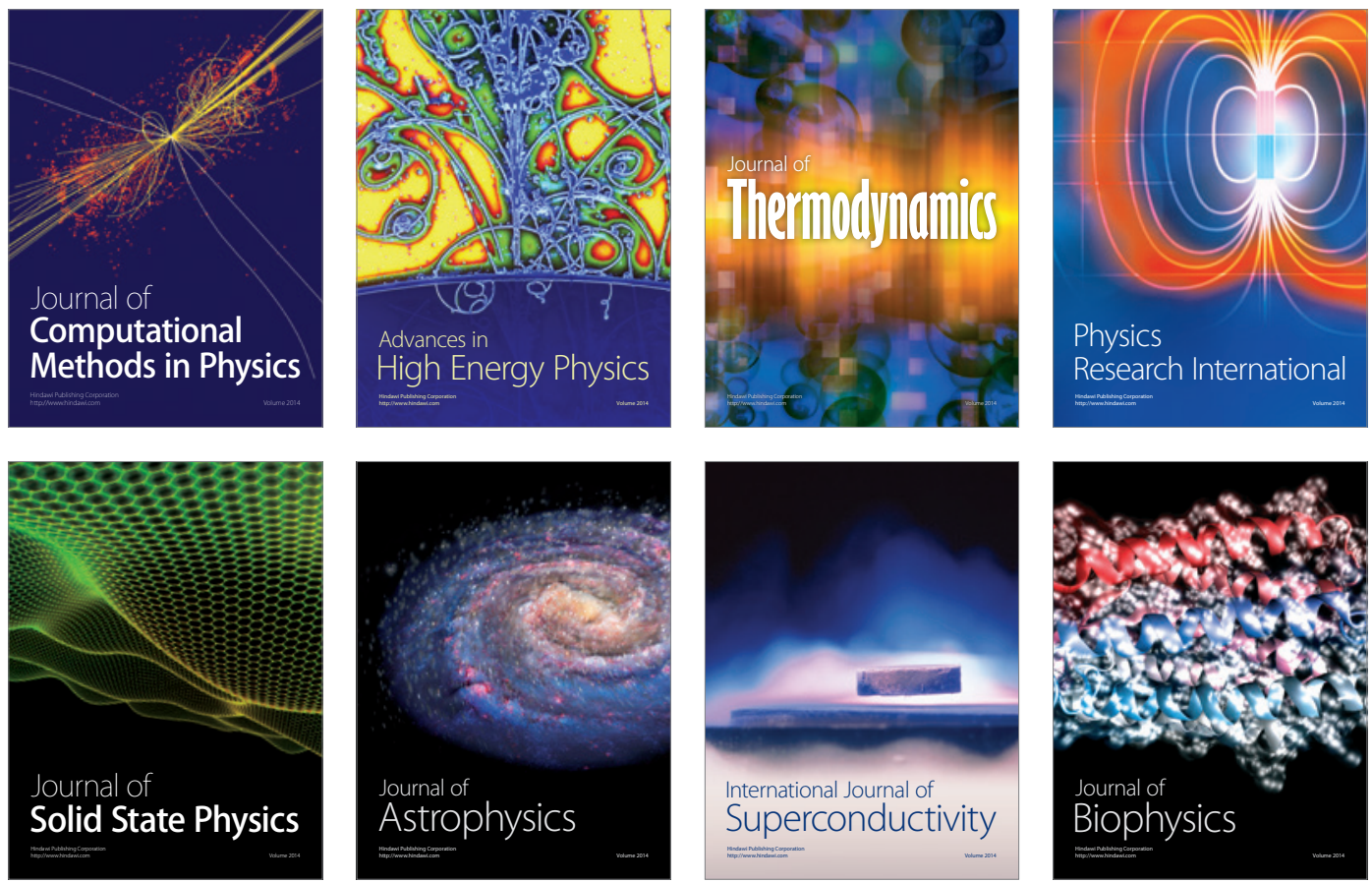
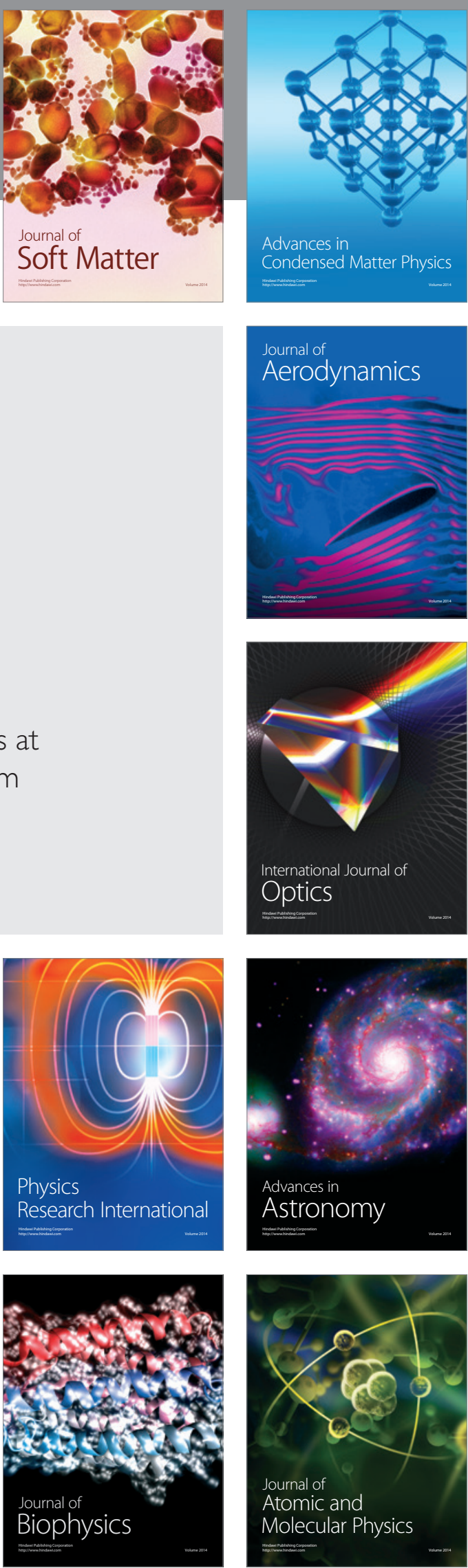